\title{
CLASH-X: A COMPARISON OF LENSING AND X-RAY TECHNIQUES FOR MEASURING THE MASS PROFILES OF GALAXY CLUSTERS
}

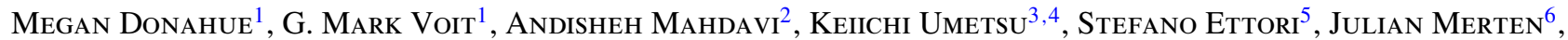 \\ Marc Postman $^{7}$, Aaron Hoffer ${ }^{1}$, Alessandro Baldi ${ }^{1}$, Dan CoE $^{7}$, Nicole Czakon $^{3,4}$, Mattias Bartelmann $^{8}$, \\ Narciso Benitez ${ }^{9}$, Rychard Bouwens ${ }^{16}$, Larry Bradley ${ }^{7}$, Tom Broadhurst ${ }^{10}$, Holland Ford ${ }^{11}$, Fabio Gastaldello ${ }^{18,23}$, \\ Claudio Grillo $^{26}$, Leopoldo Infante ${ }^{17}$, Stephanie Jouvel ${ }^{11}$, Anton Koekemoer ${ }^{7}$, Daniel Kelson ${ }^{19}$, Ofer LahaV ${ }^{12}$, \\ Doron Lemze $^{7}$, Elinor Medezinski ${ }^{7}$, Peter Melchior ${ }^{20}$, Massimo Meneghetti $^{5,6}$, Alberto Molino $^{13}$, John Moustakas $^{21}$, \\ Leonidas A. Moustakas ${ }^{6}$, Mario Nonino $^{22}$, Piero Rosati $^{14}$, Jack Sayers $^{15}$, Stella Seitz $^{24}$, ArJen Van der Wel $^{25}$, \\ WeI ZHENG ${ }^{11}$, AND AdI ZiTRIN ${ }^{15,27}$ \\ ${ }^{1}$ Physics and Astronomy Department, Michigan State University, East Lansing, MI 48824, USA; donahue@ pa.msu.edu \\ ${ }^{2}$ San Francisco State University, San Francisco, CA 94132, USA \\ ${ }^{3}$ Institute of Astronomy and Astrophysics, Academia Sinica, Roosevelt Road, Taipei 10617, Taiwan \\ ${ }^{4}$ Osservatori Astronomico di Bologna, Via Ranzani 1, I-40127 Bologna, Italy \\ ${ }^{5}$ INFN, Sezione di Bologna, viale Berti Pichat 6/2, I-40127 Bologna, Italy \\ ${ }^{6}$ Jet Propulsion Laboratory, 4800 Oak Grove Drive, Pasadena, CA 91109, USA \\ ${ }^{7}$ STScI, 3700 San Martin Drive, Baltimore, MD 21218, USA \\ ${ }^{8}$ Universität Heidelberg, Zentrum für Astronomie, Philosophenweg 12, D-69120 Heidelberg, Germany \\ ${ }^{9}$ Instituto de Astrofisica de Andalucia (CSIC), C/Camino Bajo de Huétor 24, Granada E-18008, Spain \\ ${ }^{10}$ Department of Theoretical Physics, University of the Basque Country, E-48080 Bilbao, Spain \\ ${ }^{11}$ Department of Physics \& Astronomy, The Johns Hopkins University, Baltimore, MD 21218, USA \\ ${ }^{12}$ Department of Physics \& Astronomy, University College London, Gower Street, London WC1E 6BT, UK \\ ${ }_{13}$ Instituto de Astrofisica de Andalucia, Glorieta de la Astronomia s/n, E-18008 Granada, Spain \\ ${ }^{14}$ Department of Physics and Astronomy, University of Ferrara, Via Saragat 1, I-44122 Ferrara, Italy \\ ${ }^{15}$ Department of Astronomy, California Institute of Technology, 1200 East California Boulevard, Pasadena, CA 91125, USA \\ ${ }^{16}$ Leiden Observatories, Niels Bohrweb 2, NL-2333 CA Leiden, The Netherlands \\ ${ }^{17}$ Dept Astronomía-Astrofísica, Pontificia Universidad Católica de Chile, Casilla 306, 22 Santiago, Chile \\ ${ }^{18}$ INAF-IASF, via Bassini 15, I-20133 Milan, Italy \\ ${ }^{19}$ Carnegie Observatories, 813 Santa Barbara Street, Pasadena, CA 91101, USA \\ ${ }^{20}$ Center for Cosmology and Astroparticle Physics, The Ohio State University, Columbus, OH 43210, USA \\ ${ }^{21}$ Department of Physics and Astronomy, Siena College, Loudonville, NY 12211, USA \\ 22 INAF-Osservatori Astronomico di Trieste, 34143 Trieste, Italy \\ ${ }^{23}$ University of California at Irvine, 4129 Frederick Reines Hall, Irvine, CA 92697-4575, USA \\ ${ }^{24}$ Dept. für Physik, Universitäts-Sternwarte München, Scheinerstrasse 1, D-81679 München, Germany \\ ${ }^{25}$ Max Planck Institute for Astronomy, Königstuhl 17, D-69117, Heidelberg, Germany \\ ${ }^{26}$ Dark Cosmology Centre, Niels Bohr Institute, University of Copenhagen, Juliane Maries Vej 30, DK-2100 Copenhagen, Denmark \\ Received 2014 June 2; accepted 2014 August 25; published 2014 October 1
}

\begin{abstract}
We present profiles of temperature, gas mass, and hydrostatic mass estimated from new and archival X-ray observations of CLASH clusters. We compare measurements derived from XMM and Chandra observations with one another and compare both to gravitational lensing mass profiles derived with CLASH Hubble Space Telescope and Subaru Telescope lensing data. Radial profiles of Chandra and XMM measurements of electron density and enclosed gas mass are nearly identical, indicating that differences in hydrostatic masses inferred from X-ray observations arise from differences in gas-temperature measurements. Encouragingly, gas temperatures measured in clusters by $X M M$ and Chandra are consistent with one another at $\sim 100-200 \mathrm{kpc}$ radii, but $X M M$ temperatures systematically decline relative to Chandra temperatures at larger radii. The angular dependence of the discrepancy suggests that additional investigation on systematics such as the $X M M$ point-spread function correction, vignetting, and off-axis responses is yet required. We present the CLASH-X mass-profile comparisons in the form of cosmology-independent and redshift-independent circular-velocity profiles. We argue that comparisons of circular-velocity profiles are the most robust way to assess mass bias. Ratios of Chandra hydrostatic equilibrium (HSE) mass profiles to CLASH lensing profiles show no obvious radial dependence in the $0.3-0.8 \mathrm{Mpc}$ range. However, the mean mass biases inferred from the weak-lensing (WL) and SaWLens data are different. As an example, the weighted-mean value at $0.5 \mathrm{Mpc}$ is $\langle b\rangle=0.12$ for the WL comparison and $\langle b\rangle=-0.11$ for the SaWLens comparison. The ratios of XMM HSE mass profiles to CLASH lensing profiles show a pronounced radial dependence in the $0.3-1.0 \mathrm{Mpc}$ range, with a weighted mean mass bias value rising to $\langle b\rangle \gtrsim 0.3$ at $\sim 1 \mathrm{Mpc}$ for the WL comparison and $\langle b\rangle \approx 0.25$ for the SaWLens comparison. The enclosed gas mass profiles from both Chandra and $X M M$ rise to a value $\approx 1 / 8$ times the total-mass profiles inferred from lensing at $\approx 0.5 \mathrm{Mpc}$ and remain constant outside of that radius, suggesting that $M_{\text {gas }} \times 8$ profiles may be an excellent proxy for total-mass profiles at $\gtrsim 0.5 \mathrm{Mpc}$ in massive galaxy clusters.
\end{abstract}

Key words: cosmological parameters - dark matter - galaxies: clusters: intracluster medium - gravitational lensing: strong - gravitational lensing: weak - X-rays: galaxies: clusters

Online-only material: color figures 


\section{INTRODUCTION}

CLASH (Postman et al. 2012) is a Hubble Multi-Cycle Treasury program to observe massive galaxy clusters at intermediate redshifts. It has three major scientific goals: (1) to compare the observed properties of galaxy clusters with the predictions of $\Lambda \mathrm{CDM}$ cosmology, (2) to search for galaxies at redshift $z \sim 10$ using massive clusters as gravitational lenses, and (3) to discover and monitor distant supernovae in the cluster fields through a staggered program of multi-wavelength observations. Hubble Space Telescope observations of CLASH clusters have enabled the discoveries of $z>10$ lensed galaxies (Coe et al. 2013; Zheng et al. 2012) and numerous multiply lensed galaxies at $z=4-7$ (Zitrin et al. 2012a; Balestra et al. 2013; Monna et al. 2014). CLASH has also produced strong-lensing analyses for the inner portions of individual galaxy clusters (Zitrin et al. 2012b) and combined weak-lensing (WL)/strong-lensing analyses (Umetsu et al. 2012; Coe et al. 2012; Eichner et al. 2013; Medezinski et al. 2013). The supernova survey component of CLASH discovered 39 supernova candidates and used 27 of them, discovered in the parallel fields, to measure the Type Ia supernova rate out to $z \sim 2$ (Graur et al. 2014). Moreover, three of the supernovae discovered in the prime fields were lensed by the galaxy clusters, as reported in Patel et al. (2014). Umetsu et al. (2014) describe the WL analyses of a subsample of the CLASH clusters, while Merten et al. (2014) have produced a simultaneous analysis of the strong and weak lensing of a slightly different CLASH subsample.

This paper focuses on the properties of the CLASH clusters themselves, and particularly on the cluster masses and mass profiles derived from X-ray and gravitational-lensing observations (Umetsu et al. 2014; Merten et al. 2014). According to the $\Lambda \mathrm{CDM}$ model, the gravitational influence of the invisible particles we call dark matter should produce intricate and beautiful networks of large-scale filaments with massive clusters of galaxies at the intersections. In the deep potential wells of galaxy clusters, diffuse intergalactic gas reaches temperatures sufficient to radiate X-ray light, unveiling rare and distant massive structures (e.g., Pierre et al. 2004; Gioia et al. 1990). The implications of the predominance of the hot gas in the cluster baryon budget have been known for a while (e.g., Gott \& Gunn 1971; Henry \& Arnaud 1991; David et al. 1995), but progress was slow until large X-ray surveys, especially the ROSAT All-Sky Survey, revealed hundreds of massive clusters out to redshifts $>0.5$ (e.g., Ebeling et al. 2001; Rosati et al. 1998). Many workers since have observed these clusters with Chandra and XMM-Newton to derive important cosmological constraints on dark matter, dark energy, and the baryonic mass fractions of clusters from analyses of this cluster population (Vikhlinin et al. 2009; Allen et al. 2002; Mantz et al. 2010; Ettori et al. 2009; Vikhlinin et al. 2003). All such studies require accurate calibrations of the X-ray observables (e.g., Okabe et al. 2010; Ettori 2013; Arnaud et al. 2007; Zhang et al. 2007) used to measure galaxy-cluster masses (Evrard et al. 1996).

CLASH has been testing the $\Lambda$ CDM model by measuring the radial mass profiles of clusters with gravitational-lensing observations and comparing them with the simulated profiles of $\Lambda \mathrm{CDM}$ clusters. We have found excellent agreement between the observed mass profiles (Merten et al. 2014; Umetsu et al. 2014) and those predicted from a sample of simulated clusters selected in a similar manner from a $\Lambda \mathrm{CDM}$ simulation

\footnotetext{
${ }^{27}$ Hubble Fellow.
}

(Meneghetti et al. 2014). Galaxy clusters in the CLASH sample are generally well fit by a Navarro-Frenk-White (NFW) profile (Navarro et al. 1997) with a halo concentration $c_{200} \equiv r_{\mathrm{s}} / r_{200}$, defined in terms of $r_{\mathrm{s}}$, the "scale radius" at which the local slope of the matter-density profile is $\rho \propto r^{-2}$, and $r_{200}$, the radius within which the mean density is 200 times the critical density of the universe, $\rho_{\mathrm{cr}}(z)$, at the cluster's redshift. Concentration values for CLASH clusters are typically $c_{200} \approx 4$. Both the relationship between a cluster's mass and density-profile concentration found by CLASH and the evolution of that relationship are consistent with $\Lambda \mathrm{CDM}$ simulations (Merten et al. 2014; Meneghetti et al. 2014).

Here we take advantage of the unparalleled gravitationallensing data collected by the CLASH collaboration to assess the level of agreement between the cluster mass profiles inferred from X-ray observations and those measured through lensing. Gravitational lensing is considered the gold standard for cluster mass measurements because of its lack of sensitivity to cluster astrophysics (Tyson et al. 1990; Kaiser \& Squires 1993). However, lensing measurements suffer from intrinsic scatter owing to statistical fluctuations in the amount of matter along the line of sight to the cluster but outside of the cluster itself (Dalal et al. 2005; King \& Corless 2007). In this paper, as in most cosmological studies of galaxy clusters, we will define the outer boundary of a cluster as the radius $r_{\Delta}$ of a sphere encompassing a mean matter density $\Delta \rho_{\mathrm{cr}}$. Projected matter fluctuations outside of this radius do not significantly bias the cluster mass measurements from gravitational lensing but are expected to lead to significant $(\sim 20 \%)$ scatter between lensinginferred masses and spherical-overdensity masses (Metzler et al. 2001; Hoekstra 2001, 2003; Becker \& Kravtsov 2011).

Cluster mass measurements inferred from X-ray observations are believed to have less statistical scatter than those inferred from lensing but are subject to greater astrophysical uncertainties, which have the potential to introduce systematic bias. This paper focuses on mass measurements invoking the assumption that the intracluster medium is in hydrostatic equilibrium (HSE), but not all X-ray studies adopt that assumption. Some rely on the relation between total mass and the best-fitting spectroscopically determined gas temperature $T_{X}$, which can be calibrated with either numerical simulations or lensing observations (Horner et al. 1999; Finoguenov et al. 2001; Xu et al. 2001; Ettori et al. 2002; Vikhlinin et al. 2006). Others use total gas mass $M_{\text {gas }}$ or the quantity $Y_{X}=T_{X} \times M_{\text {gas }}$ as mass proxies (Nagai et al. 2007; Arnaud et al. 2010; Okabe et al. 2010; Mahdavi et al. 2013).

So why bother with hydrostatic methods, given that galaxy clusters are unlikely to be in perfect HSE? One answer is that HSE mass estimates are relatively easy to determine for galaxy clusters with a single central peak in X-ray surface brightness, as long as the data are sufficient to generate radial profiles of gas temperature $T_{\mathrm{gas}}$ and electron density $n_{e}$. $\mathrm{X}$-ray observatories accomplish this task by collecting X-ray photons while recording each event's energy and point of origin in the sky. Photon events then can be compiled into both two-dimensional maps of X-ray emission and one-dimensional spectra of specific regions on the sky, from which $T_{X}(r)$ and $n_{e}(r)$ can be determined. Another answer is that many of the clusters produced in numerical simulations are not far from equilibrium. By the late 1990s, cluster simulations of gas outside of the cooling core $(r \gtrsim 100 \mathrm{kpc})$ could generate fairly reliable representations of cluster X-ray observations. Those same simulations predicted that nonthermal motions, such as 
turbulence and bulk motions remaining in the gas after interactions and mergers, provide on average only $10 \%-20 \%$ of the pressure support in the intracluster gas (Evrard 1990). The timescale for intracluster gas to respond to changes in the cluster potential is $\sim T_{8}^{-1 / 2} r_{\mathrm{Mpc}} \mathrm{Gyr}$, where $T_{8}=T_{\mathrm{gas}} / 10^{8} \mathrm{~K}$ and $r_{\mathrm{Mpc}}$ is the cluster radius in megaparsecs. Incomplete thermalization of residual motions from a merger event may contribute significant pressure support shortly after a merger, but the cluster subsequently relaxes toward HSE on a $\sim 1$ Gyr timescale.

While a cosmologist might be concerned about time-varying offsets between a cluster's HSE mass and its actual mass, a true astrophysicist finds them interesting, because the difference between those masses reflects the thermalization state of gas motions in the intracluster medium and the speed at which they damp. (See Reiprich et al. (2013) for a review of cluster outskirts.) For example, the presence of long-lived turbulence in a cluster would indicate that the gas has relatively low viscosity, a hydrodynamical property of the cluster gas that is currently not well constrained by observations (Dolag et al. 2005; Sijacki $\&$ Springel 2006). Measuring systematic differences between the HSE mass of a cluster and the "true" mass measured by gravitational lensing, also known as the X-ray mass bias $b_{X}=1-M_{\mathrm{HSE}} / M_{\text {true }}$ (Vikhlinin et al. 2006; Evrard et al. 2008), therefore provides valuable information about the physics of the intracluster medium. Theoretical simulations generally predict $\left\langle b_{X}\right\rangle \approx 0.2$ for an unbiased population of clusters (e.g., Rasia et al. 2012; Nelson et al. 2014). On a cluster-by-cluster basis, the assumption of spherical symmetry produces scatter in the $b_{X}$ measurement, because departures from symmetry (or triaxiality) affect the lensing mass estimate more than the X-ray mass estimate (cf. Coe et al. 2012). Lensing masses have measurement uncertainties of their own, and current estimates of the systematic uncertainties range from about $15 \%-20 \%$ for discrepant measurements for the same cluster from different groups to $8 \%$ for internal systematics for the CLASH WL estimates (Umetsu et al. 2014).

Lately, interest in the value of $\left\langle b_{X}\right\rangle$ has heightened because of the discrepancy between the cosmological parameters inferred from Planck observations of the primary anisotropies of the cosmic microwave background (CMB) and the galaxy-cluster counts provided by Planck observations of the Sunyaev-Zeldovich (SZ) effect (Planck Collaboration et al. $2013 b$ ). According to the $\Lambda$ CDM model, the number density of galaxy clusters of a given mass at a given redshift depends sensitively on the matter-density parameter $\Omega_{M}$ and the matterperturbation amplitude $\sigma_{8}$. Relating $\Lambda \mathrm{CDM}$ cluster predictions to SZ cluster counts currently requires an assumption about mass bias because there is no definitive calibration of the relationship between SZ signal and cluster mass. Instead, masses determined from X-ray observations with the $X M M$ satellite were used to establish this relationship (Arnaud et al. 2010), but using the $\left\langle b_{X}\right\rangle \approx 0.2$ value found in simulations in that calibration leads to an overprediction of SZ counts. A larger mass bias corresponding to $\left\langle b_{X}\right\rangle \approx 0.4$ can reconcile the SZ counts with the Planck CMB cosmology (Planck Collaboration et al. 2013c). Alternatively, streaming of neutrinos with a mass sum $\sim 0.5 \mathrm{eV}$ can suppress the cosmic perturbations on galaxycluster scales enough to explain the discrepancy (Hamann \& Hasenkamp 2013; Wyman et al. 2014; Battye \& Moss 2014), but this mass value is in tension with other neutrinomass constraints from large-scale structure. Either way, accurate measurements of mass bias will be critical for resolving this issue.
In another joint analysis of CMB results and cluster properties, Rozo et al. (2014) derive an $L_{X}-M$ relation that reconciles the thermal SZ power spectrum from WMAP7 with cluster scaling relations between richness and mass and the SZ signal. They show rough consistency of this relation with published values $M_{500}$ for two CLASH clusters and make predictions based on $L_{X}$ for the masses of a subset of the CLASH clusters.

Our discussion of these topics proceeds like this: Section 2 introduces the CLASH cluster sample. Section 3 describes the $\mathrm{X}$-ray data analysis. Section 4 presents the radial profiles of gas density and gas temperature, on which the rest of the analysis is based, and calls attention to a systematic radially dependent discrepancy between the temperatures measured with $X M M$ and those measured with Chandra. Section 5 shows the individual cluster mass profiles as plots of circular velocity $v_{\text {circ }} \equiv[G M(r) / r]^{1 / 2}$ as a function of radius and encourages others to provide mass profiles in this form, in order to minimize dependences on cosmological assumptions. Section 6 compares the Chandra mass profiles with the CLASH lensing profiles; Section 7 does the same for the $X M M$ profiles, showing that the $X M M$ mass bias at large radii may be as great as $\left\langle b_{X}\right\rangle \approx 0.4$. Section 8 shows that gas masses derived from both Chandra and $X M M$ closely agree and are quite close to $1 / 8$ of the lensing mass outside of $\sim 0.5 \mathrm{Mpc}$, implying that $8 M_{\text {gas }}$ is a good approximation to the total enclosed mass within large radii for massive clusters. Section 9 discusses the implications of our results for the Planck cluster-mass discrepancy, and Section 10 summarizes our findings. When necessary, we adopt a vanilla $\Lambda$ CDM cosmology with a single decimal place $\left(\Omega_{\mathrm{M}}=0.3\right.$, $\Omega_{\Lambda}=0.7$, and $H_{0}=70 h_{70} \mathrm{~km} \mathrm{~s}^{-1} \mathrm{Mpc}^{-1}$ ), but we prefer to state results in forms that depend as little as possible on cosmological assumptions.

\section{THE CLASH CLUSTER SAMPLE}

The CLASH program and strategy are completely described in Postman et al. (2012). All 25 clusters are high-mass, hightemperature $\left(T_{X} \gtrsim 6 \mathrm{keV}\right)$ clusters of galaxies. These 25 galaxy-cluster targets fall into two general categories. Twenty were chosen for their relatively symmetric X-ray appearance, primarily to test the $\Lambda \mathrm{CDM}$ predictions for radial mass profiles. Five are "high-magnification" clusters chosen for the size of their Einstein radius, primarily to maximize the probability of finding highly magnified $z>10$ background galaxies. Table 1 lists all 25 CLASH clusters, with the high-magnification subset at the bottom, and indicates the data included in this paper for each cluster. The CLASH WL result is based on a joint shear and magnification analysis of data sets primarily from Subaru and is described in Umetsu et al. (2014). The CLASH joint modeling of strong- and weak-lensing-SaWLens-data from both Hubble and ground-based data for a subsample of CLASH clusters is described in Merten et al. (2014). Both lensing mass measurements are based on spherical NFW fits to the projected mass density profiles $\Sigma(R)$ recovered from the respective data sets. These features distinguish these analyses from the majority of previous lensing mass measurements, which are mostly based on tangential shear fitting.

Archival Chandra X-ray data were available for all the CLASH clusters at the start of the project, because those observations were used to select the targets. In this regard, the CLASH collaboration is indebted to many other X-ray observers, and especially the Massive Cluster Survey (MACS) led by Harald Ebeling (Ebeling et al. 2001). That survey 
Table 1

CLASH Cluster Data

\begin{tabular}{|c|c|c|c|c|c|c|c|c|c|}
\hline Name & R.A. (J2000) & Decl. (J2000) & $z$ & Hi-Maga & $K_{0}^{\mathrm{b}}$ & Chandra & $X M M$ & Subaru $\mathrm{WL}^{\mathrm{c}}$ & SaWLens $^{\mathrm{d}}$ \\
\hline A209 & $01: 31: 52.54$ & $-13: 36: 40.4$ & 0.206 & & 105.5 & $\mathrm{Y}$ & $\mathrm{Y}$ & $\mathrm{Y}$ & $\mathrm{Y}$ \\
\hline A383 & $02: 48: 03.40$ & $-03: 31: 44.9$ & 0.187 & & 13.0 & $\mathrm{Y}$ & $\mathrm{Y}$ & $\mathrm{Y}$ & $\mathrm{Y}$ \\
\hline MACS J0329-02 & $03: 29: 41.56$ & $-02: 11: 46.1$ & 0.450 & & 11.1 & $\mathrm{Y}$ & & $\mathrm{Y}$ & Y \\
\hline MACS J0429-02 & $04: 29: 36.05$ & $-02: 53: 06.1$ & 0.399 & & 17.2 & $\mathrm{Y}$ & $\mathrm{Y}$ & $\mathrm{Y}$ & $\mathrm{Y}$ \\
\hline MACS J0744+39 & $07: 44: 52.82$ & $+39: 27: 26.9$ & 0.686 & & 42.4 & $\mathrm{Y}$ & $\mathrm{Y}$ & $\mathrm{Y}$ & $\mathrm{Y}$ \\
\hline A611 & 08:00:56.82 & $+36: 03: 23.6$ & 0.288 & & 125 & $\mathrm{Y}$ & & $\mathrm{Y}$ & $\mathrm{Y}$ \\
\hline MACS J1115+01 & $11: 15: 51.90$ & $+01: 29: 55.1$ & 0.355 & & 14.8 & $\mathrm{Y}$ & $\mathrm{Y}$ & $\mathrm{Y}$ & Y \\
\hline A 1423 & $11: 57: 17.36$ & $+33: 36: 37.5$ & 0.213 & & 68.3 & $\mathrm{Y}$ & & & \\
\hline MACS J1206-08 & 12:06:12.09 & $-08: 48: 04.4$ & 0.439 & & 69.0 & $\mathrm{Y}$ & $\mathrm{Y}$ & $\mathrm{Y}$ & $\mathrm{Y}$ \\
\hline CL J1226+3332 & $12: 26: 58.25$ & $+33: 32: 48.6$ & 0.890 & & 166 & $\mathrm{Y}$ & $\mathrm{Y} / \mathrm{N}^{\mathrm{e}}$ & & $\mathrm{Y}$ \\
\hline MACS J1311-03 & $13: 11: 01.80$ & $-03: 10: 39.8$ & 0.494 & & 47.4 & $\mathrm{Y}$ & & & $\mathrm{Y}$ \\
\hline RX J1347-1145 & $13: 47: 30.62$ & $-11: 45: 09.4$ & 0.451 & & 12.5 & $\mathrm{Y}$ & $\mathrm{Y}$ & $\mathrm{Y}$ & $\mathrm{Y}$ \\
\hline MACS J1423+24 & $14: 23: 47.88$ & $+24: 04: 42.5$ & 0.545 & & 10.2 & $\mathrm{Y}$ & & & $\mathrm{Y}$ \\
\hline MACS J1532+30 & $15: 32: 53.78$ & $+30: 20: 59.4$ & 0.362 & & 16.9 & $\mathrm{Y}$ & $\mathrm{Y}$ & $\mathrm{Y}$ & $\mathrm{Y}$ \\
\hline MACS J1720+35 & $17: 20: 16.78$ & $+35: 36: 26.5$ & 0.387 & & 94.4 & $\mathrm{Y}$ & & $\mathrm{Y}$ & $\mathrm{Y}$ \\
\hline A2261 & $17: 22: 27.18$ & $+32: 07: 57.3$ & 0.224 & & 61.1 & $\mathrm{Y}$ & $\mathrm{Y}$ & $\mathrm{Y}$ & $\mathrm{Y}$ \\
\hline MACS J1931-26 & $19: 31: 49.62$ & $-26: 34: 32.9$ & 0.352 & & 14.6 & $\mathrm{Y}$ & $\mathrm{Y}$ & $\mathrm{Y}$ & $\mathrm{Y}$ \\
\hline RX J2129+0005 & $21: 29: 39.96$ & $+00: 05: 21.2$ & 0.234 & & 21.1 & $\mathrm{Y}$ & $\mathrm{Y}$ & $\mathrm{Y}$ & Y \\
\hline MS 2137-2353 & 21:40:15.17 & $-23: 39: 40.2$ & 0.313 & & 14.7 & $\mathrm{Y}$ & $\mathrm{Y}$ & $\mathrm{Y}$ & $\mathrm{Y}$ \\
\hline RXC J2248-4431 & $22: 48: 43.96$ & $-44: 31: 51.3$ & 0.348 & & 42.2 & $\mathrm{Y}$ & $\mathrm{Y}$ & $\mathrm{Y}$ & $\mathrm{Y}$ \\
\hline MACS J0416-24 & 04:16:08.38 & $-24: 04: 20.8$ & $0.397^{\mathrm{f}}$ & $\mathrm{Y}$ & 400 & $\mathrm{Y}$ & & $\mathrm{Y}$ & \\
\hline MACS J0647+70 & $06: 47: 50.27$ & $+70: 14: 55.0$ & 0.584 & $\mathrm{Y}$ & 225 & $\mathrm{Y}$ & $\mathrm{Y}$ & $\mathrm{Y}$ & \\
\hline MACS J0717+37 & $07: 17: 32.63$ & $+37: 44: 59.7$ & 0.548 & $\mathrm{Y}$ & 220 & $\mathrm{Y}$ & $\mathrm{Y}$ & $\mathrm{Y}$ & \\
\hline MACS J1149+22 & $11: 49: 35.69$ & $+22: 23: 54.6$ & 0.544 & $\mathrm{Y}$ & 280 & $\mathrm{Y}$ & & $\mathrm{Y}$ & \\
\hline MACS J2129-07 & $21: 29: 26.06$ & $-07: 41: 28.8$ & 0.570 & $\mathrm{Y}$ & 200 & $\mathrm{Y}$ & & & \\
\hline
\end{tabular}

Notes.

${ }^{a}$ High-Mag of Y indicates that the cluster is one of the five CLASH clusters selected for their lensing properties.

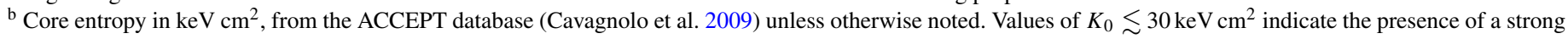
cool core.

${ }^{c}$ Umetsu et al. 2014. Note: the weak-lensing data for RX J2248-4431 is from the $2.2 \mathrm{~m}$ Wide Field Imager ESO/Chile (Gruen et al. 2013).

d Merten et al. 2014.

e $X M M$ data exist for this cluster and were analyzed for CLASH-X but are not of sufficient quality for comparisons with the other data sets.

${ }^{\mathrm{f}}$ Redshift for MACS 0416-24 from Postman et al. (2012) is updated by Ebeling et al. (2014).

originally identified many of the clusters studied here and collected much of the Chandra X-ray data. All of the archival data were sufficient for deriving electron density and gas temperature in a minimum of three radial bins, and we have previously provided independent analyses of many of those data sets in the ACCEPT database Cavagnolo et al. (2009).

A majority of the CLASH clusters also had archival $X M M$ data, but more than a few of those data sets turned out to be highly contaminated by flares and are unusable for this project. We therefore acquired new XMM data for six CLASH clusters, A2261, MACS 1931, MACS 1115, MACS 0429, MACS 1720, and MACS 1423, and present those data in this paper. The latter two data sets, especially for MACS 1423, were severely compromised by flares, but they still represent independent $\mathrm{X}$-ray measurements. $X M M$ observations are particularly desirable because of its larger collecting area and field of view, which allow us to extend the X-ray hydrostatic mass profiles out to $r_{500}$, increasing the radial range in which the X-ray and WL data sets overlap.

Table 2 lists both the Chandra and XMM data sets used in this paper, along with the flare-free exposure times available in each data set. We did not utilize all of the observations available in the archive for each cluster because the flare contamination could be considerable for some of these data sets, and for others, the gain achieved by adding an incremental amount of exposure was not worth the added systematic uncertainty of potential calibration variance. In general, if a single data set added fewer than $30 \%$ to the total counts, we did not use it.

\section{X-RAY DATA ANALYSIS}

Derivations of HSE mass profiles from X-ray data entail a two-step process. First, one must prepare the data for fitting by selecting a cluster center and dividing the X-ray photon events among a series of concentric annular bins. Then one must fit the binned two-dimensional data with a projected threedimensional (3D) model, usually a spherically symmetric one. This section describes the two-step process used to derive the radial profiles presented here. We use identical procedures to fit both the Chandra and XMM data, so that the comparisons we make can be as direct as possible. Our primary fitting tool is the Joint Analysis of Cluster Observations (JACO) code (Mahdavi et al. 2007, 2013), which we describe at the end of the section. Experienced X-ray observers may find some parts of this section rather basic. We will attempt to paint a complete picture that allows astronomers outside the X-ray community to see where possible systematic differences between the Chandra and XMM results can arise.

\subsection{Chandra Data Preparation}

We reprocessed all of the Chandra data identified in Table 1 with CIAO 4.6.1 (released 2014 February) and CALDB 
Table 2

X-Ray Observations

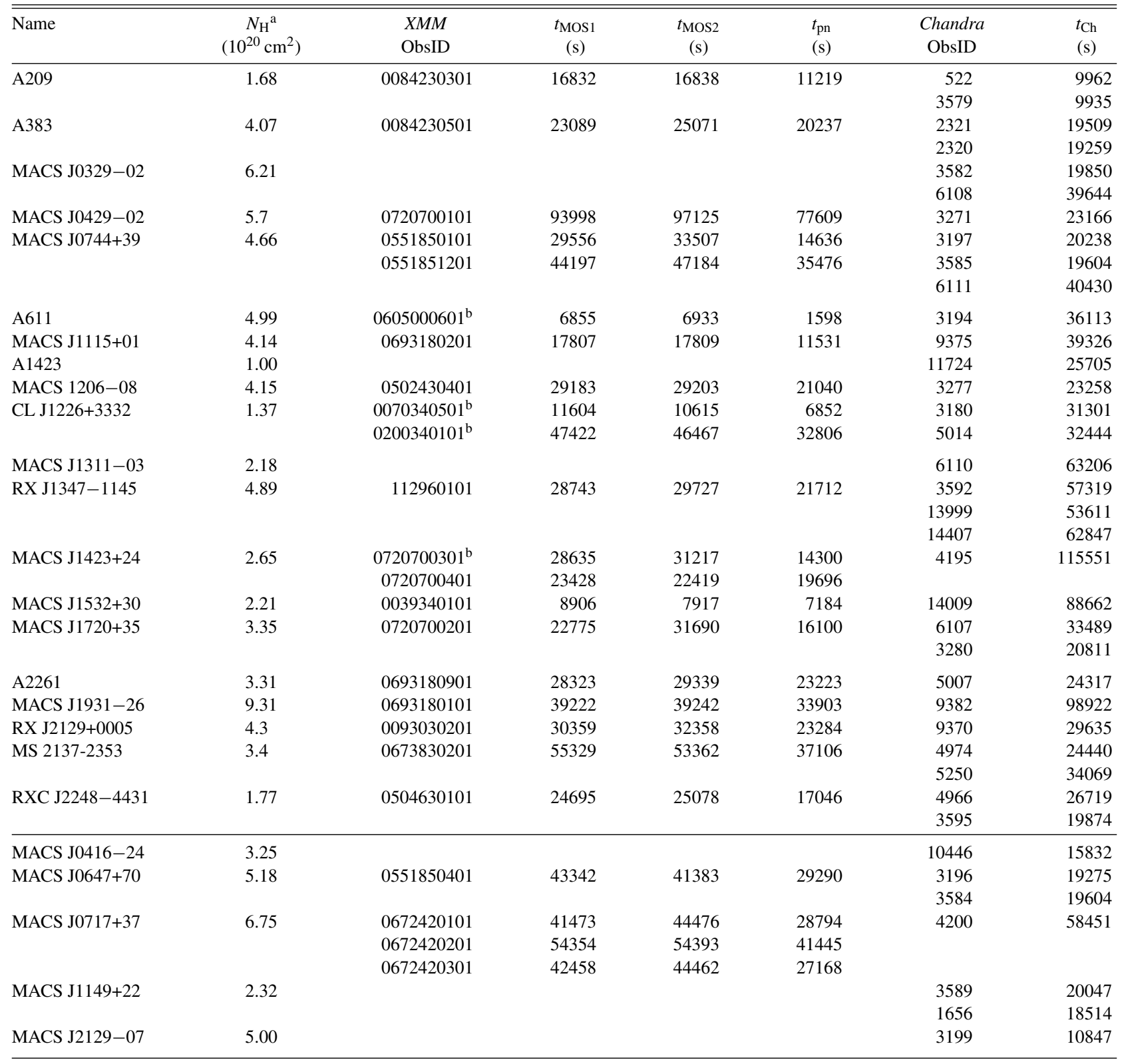

Notes.

${ }^{\text {a }}$ Soft X-ray absorbing galactic hydrogen column density along the line of sight to the cluster.

b These $X M M$ data were insufficient to define an independent temperature/mass profile.

4.5.9 (released 2013 November). The version and date of the calibration are important to note because this particular calibration revision introduced some important corrections to the soft energy response due to contamination issues. Time-dependent contamination means that everything from the energy response to the gain has a time dependence. Calibration changes and updates therefore may introduce differences between today's results and results published in the past.

In order to remove flares from the data, light curves limited to events between 0.5 and $7.0 \mathrm{keV}$ were extracted from source-free areas of the detectors. Any time interval with an event rate $3 \sigma$ above the mean event rate was then excluded from the timeline. Particularly serious flares were excluded from the timeline using a more conservative $2 \sigma$, which effectively removes the ramp-up and ramp-down times near a large flare and the short intervals between major flares.

Most of the CLASH Chandra data sets were free of significant flaring. The "deflare" script in CIAO was used to generate lists of good time intervals, which were then applied to remove events acquired during flares from the events files. The majority of these data were taken using "VFAINT" mode, which allows for a stricter cleaning of background events at the expense of only $1 \%-2 \%$ of the source counts.

Bright point sources were identified and removed using the CIAO detection algorithm wavdetect together with a map of the point-spread function (PSF) size as a function of location on the detector. We filtered regions around these point sources from the event lists. For Chandra data, point-source excision 
did not result in a significant loss of cluster event counts or sky area coverage because the point sources are quite compact.

Backgrounds coming from fainter X-ray point sources, soft $\mathrm{X}$-ray emission from hot gas in our own Galaxy, and nonflaring particle events must be accounted for using deep background files. Each data set was therefore matched to a deep background file from a similar observation epoch courtesy of the Chandra data center (Hickox \& Markevitch 2007; Markevitch et al. 2003). These deep-background files, which include both particle and photon backgrounds, were filtered and reprojected to match the target observations. As recommended by the Chandra Science Center, we rescaled the reprojected background rates to match the observation count rates between 10.0 and $12.0 \mathrm{keV}$, an energy regime nearly completely dominated by high-energy particle events, not photons. The mechanism we used was to adjust the effective exposure time in the headers of the reprojected background event files. Typically this rescaling affected the effective background exposure time by less than $10 \%-15 \%$.

Annular bin boundaries are then selected so that each annular bin contains at least 1500 counts of photon signal from the cluster. In some cases, these counts come from multiple observations of the same cluster. Each annular bin has a minimum radial width at least a few times the PSF width. Bins that are too narrow can cause the fitting of a 3D model to become unstable, because deprojection and PSF correction have some mutual covariance.

Once the annular bins are selected, we generate X-ray spectra for each bin from both the image files and the deep background files. For Chandra, spectra were created with the CIAO specextract script. Preparation of X-ray spectra includes the computation of individual weighted redistribution matrix files and ancillary response files. We extracted spectra binned in energy from 0.5 to $11.0 \mathrm{keV}$, with a bin width of $38 \mathrm{eV}$.

At this point the annular spectra are ready for fitting, but with one caveat. The positional variation of the galactic soft $\mathrm{X}$-ray background is not accounted for by our use of the deepbackground files. Therefore, when fitting any of these data sets, we allow for a possible over- or undersubtraction of the galactic soft X-ray background by fitting the contribution of a uniform soft thermal X-ray background (fixed at solar abundance), parameterized by its normalization (positive or negative) and temperature. (Mahdavi et al. 2007). Fixing the temperature of this component at $0.5 \mathrm{keV}$ did not affect the outcome.

\subsection{XMM Data Preparation}

Our preparation of the $X M M$ data proceeds along much the same lines. We reprocess the $X M M$ Observation Data Files using $X M M$ Science Analysis System tasks (version xmmsas_20120621_1331) and current calibration files. The most recently acquired data were processed using version xmmsas_20131209_1901-13.5.0. We used data from all three spectroscopic-imaging telescope/detector combinations on $X M M$ for the European Photon Imaging Camera: Metal Oxide Semiconductor CCD cameras 1 and 2 (MOS1 and MOS2), and a third, back-illuminated CCD camera called the pn. We filtered the event light curves using standard $X M M$ criteria and procedures described in Mahdavi et al. (2013).

Similarly to the Chandra processing, we exclude bright point sources, using the CIAO task wavdetect to locate sources on the pn-detector image. We always visually inspect the sources identified on this list to make sure not to exclude the cluster itself. Occasionally, additional sources are added manually to this list to remove noise spikes near chip boundaries and other sources of excess counts that wavdetect missed. Usually no more than a few additional excision regions are defined by hand. We use relatively source-free regions of the MOS1, MOS2, and pn detectors to assess the particle background between 10 and $12 \mathrm{keV}$. To do this, we calculate the $10-12 \mathrm{keV}$ count rate in the blank-sky fields provided by the $X M M$ Science Operations Center (Carter \& Read 2007). The ratio of the blanksky $10-12 \mathrm{keV}$ count rate to the observation 10-12 keV count rate is used to normalize the spatially resolved blank-sky spectra, which are then subtracted from source spectra prior to any fitting. While this procedure mitigates the particle background, it has the side effect of over- or undersubtracting the astrophysical background (i.e., the contribution of unresolved active galactic nuclei and the galactic soft X-ray background). To address this side effect, as with the Chandra spectra, we include nuisance background models that are uniform over the field of view of the observations. These backgrounds are modeled as thermal plasmas with adjustable temperature and, for this work, fixed solar metallicity, with positive or negative normalization specific to each detector. Therefore, a total of five nuisance parameters for the case of full XMM usage (MOS1+MOS2+pn) are used to mitigate this residual background.

For $X M M$ data we employ the techniques described in Mahdavi et al. (2013) to prepare the spectra for fitting, which includes the subtraction of the "out-of-time" events from the $X M M$ pn spectra. (Out-of-time events, which are events that are misassigned locations along the readout because they arrive during the short time a detector is read out, are negligible for the MOS and for all but the brightest sources for Chandra.) As with the Chandra data, we choose annular bin boundaries so that each bin contains at least 1500 source events and a minimum width of $8^{\prime \prime}$, and we include nuisance parameters in the fitting procedure to account for direction-dependent differences in the temperature and normalization of the galactic soft X-ray background.

\subsection{A JACO Primer}

Our primary tool for deriving gas and HSE mass profiles from both Chandra and XMM data is JACO (Mahdavi et al. 2007), which can provide simultaneous fits to X-ray, SZ, and WL data. Here we use it to fit only the X-ray data. JACO employs parametric models for both the dark matter density and gas density in the fitting procedure. In CLASH-X, the dark matter profiles are assumed to have a spherically symmetric NFW form, parameterized by a scale radius $r_{\mathrm{s}}$ and a density normalization $\rho_{\mathrm{s}}$ at that radius, so that

$$
\rho_{\mathrm{DM}}(r)=\frac{4 \rho_{\mathrm{s}}}{\left(r / r_{\mathrm{s}}\right)\left[1+\left(r / r_{\mathrm{s}}\right)\right]^{2}},
$$

whereas the gas-density profiles are modeled with a more flexible triple $\beta$-model, with one component multiplied by a radial power law of index $-\alpha$ :

$$
\begin{aligned}
n_{e}(r)= & n_{e 0}\left(\frac{r}{r_{0}}\right)^{-\alpha}\left(1+\frac{r}{r_{e 0}}\right)^{-3 \beta_{0} / 2}+n_{e 1}\left(1+\frac{r}{r_{e 1}}\right)^{-3 \beta_{1} / 2} \\
& +n_{e 2}\left(1+\frac{r}{r_{e 2}}\right)^{-3 \beta_{2} / 2} .
\end{aligned}
$$

If the surface brightness profile is adequately fit by a single $\beta$-model truncated by a power law (i.e., the first term), we set 
$n_{e 1}=n_{e 2}=0$. JACO can also allow for a stellar contribution to the total mass-density profile in the form of an Einasto profile, but that feature is not used here. We do, however, allow for a radial metallicity dependence in the intracluster medium, with a profile

$$
\frac{Z}{Z_{\odot}}=Z_{0}\left(1+\frac{r^{2}}{r_{Z}^{2}}\right)^{-3 \beta_{Z}}
$$

Using this combined parametric model, JACO can compute the projected X-ray spectrum coming from each line of sight through the target cluster by making a strong assumption: the radial gas-temperature profile is determined by requiring that the gas be in HSE in the combined potential well of the dark matter and gas. In this paper, we will refer to the temperature determined in this way as $T_{\mathrm{JACO}}$.

Once JACO has made a parametric cluster model, it generates a synthetic event spectrum for each annular bin by convolving the cluster model with an energy-dependent instrumental PSF and adding a background model. As described in the data preparation subsections, we subtract the particle spectrum using renormalized deep background fields and account for the overor undersubtraction of the galactic soft X-ray background by fitting it to a parameterized soft thermal component. These corrections are part of the JACO fitting procedure.

Correction for the instrumental PSF turns out to be critical for the CLASH-X mass-profile comparison, because the cores of galaxy clusters are far brighter than the regions at $r_{2500}$ and $r_{500}$, where we would like to make comparisons with the WL data. Proper PSF correction is much more important for $X M M$ than Chandra because its half energy width (HEW) of its PSF is so much broader $\left(13^{\prime \prime}-17^{\prime \prime}\right.$ for $X M M^{28}$ compared to $<1^{\prime \prime}$ on axis for Chandra, ${ }^{29}$ according to the instrument handbooks). We have compared JACO results with and without PSF correction and find that best-fit masses and temperatures derived from uncorrected models are systematically lower than those for corrected models. At least part of this effect comes from the scattering of X-ray photons from the cooler, brighter cores of some clusters to larger radii in the detector, thereby reducing the best-fitting temperatures at those radii. (See, for example, Maxim Markevitch's white paper on A1835, posted on the Chandra calibration Web site. ${ }^{30}$ ) But those scattering events should be accounted for by the PSF correction procedure. The results we present in Section 4.2 suggest that additional $X M M$ PSF corrections might be necessary to bring the $X M M$ and Chandra results into acceptable agreement.

JACO obtains constraints on all parameters of each cluster model through a Monte Carlo Markov Chain (MCMC) procedure that produces likelihood distributions for all the radial profiles of interest. The uncertainty ranges on X-ray HSE mass profiles reported in Section 5 come from this MCMC procedure and represent $68 \%$ confidence intervals. This "forward-fitting" procedure fundamentally differs from the "deprojection" procedure commonly used in X-ray astronomy, which obtains radial temperature and density profiles by sequentially fitting and then subtracting the contribution of each spherical shell, starting from the outermost part of the cluster and finishing in the core (e.g., McLaughlin 1999; Croston et al. 2006). Instead, JACO fits all shells simultaneously, obtaining stronger constraints on the

\footnotetext{
28 http://xmm.esac.esa.int/external/xmm_user_support/documentation/ uhb/onaxisxraypsf.html

29 http://asc.harvard.edu/proposer/POG/html/chap4.html

30 http://arxiv.org/abs/astro-ph/0205333
}

properties of each shell (which are correlated) at the expense of imposing a parametric model on the overall mass distribution.

We have tested the JACO cluster fits in two different ways. We have compared them with electron density and pressure profiles from ACCEPT (Cavagnolo et al. 2009) and with results from the deprojection code used in Ettori et al. (2010), and we find good agreement. We have also used JACO to produce nonparametric fits for each cluster to a model in which concentric shells with the same inner and outer radii as the annular bins contain isothermal gas of uniform density. This is not a "deprojection" but rather an MCMC fit in which the gas density and gas temperature of each bin are the fitted parameters. It is not subject to the assumptions of HSE and NFW-ness of the mass profile and can therefore be used to check whether those assumptions distort the gas density and temperature profiles derived in the parametric fitting procedure. The results have greater uncertainties but are statistically consistent with those obtained from the parametric fits.

For both $X M M$ and Chandra spectra, the raw spectrum energy bin was $38 \mathrm{eV}$. We typically limited the spectral fit to $0.7-8.0 \mathrm{keV}$, but we occasionally truncated the fits at $7.0 \mathrm{keV}$. Energy bins were grouped to a minimum of 25 counts per grouped spectral bin. We conservatively restrict the fits to radial bins where the signal-to-total counts ratio exceeds 0.25 , equivalent to the signal-to-background ratio threshold of 0.3 recommended by Leccardi \& Molendi (2008) to avoid any strong systematics in the background treatment. All spectra and JACO configuration files are provided in the CLASH public data products site hosted by MAST. ${ }^{31}$ Table 3 contains information about the JACO parameterized fit to the full cluster data set for Chandra and, where available, XMM data.

\section{PROFILES OF GAS PROPERTIES}

This section presents the gas properties derived from the JACO fits. We find very good agreement between the electrondensity profiles coming from the Chandra and XMM data sets, and therefore very good agreement between the gasmass profiles as well. However, agreement between the gastemperature profiles is not as satisfactory. Temperature agreement between Chandra and XMM is good in the cluster cores $(\lesssim 200 \mathrm{kpc})$ but becomes progressively worse at larger radii, with $T_{\mathrm{JACO}}(\mathrm{xmm}) \approx 0.75 T_{\mathrm{JACO}}$ (chandra) at $r \sim 800 \mathrm{kpc}$, indicating either a need for more accurate $X M M$ PSF correction, improved knowledge of the off-axis response, or a background-subtraction issue with either XMM or Chandra.

\subsection{Gas Density and Gas Mass}

The left panel of Figure 1 shows the ratio between $X M M$ derived gas density and Chandra-derived gas density as a function of radius for each CLASH-X cluster with both XMM and Chandra data, along with the unweighted mean, weighted mean, and median ratios at each radius. Here and throughout the paper, we present geometric means instead of arithmetic means for these ratios, so that the means are symmetric with respect to an exchange of the numerator and denominator (i.e., $\langle A \mid B\rangle=\langle B / A\rangle^{-1}$ for geometric means but not arithmetic means). Individual clusters show some density-ratio excursions arising from the fitting procedure, because we are fitting parametric electron-density profiles to Chandra and XMM data sets with different bin spacing. However, the mean ratios are

\footnotetext{
31 http://archive.stsci.edu/prepds/clash/
} 

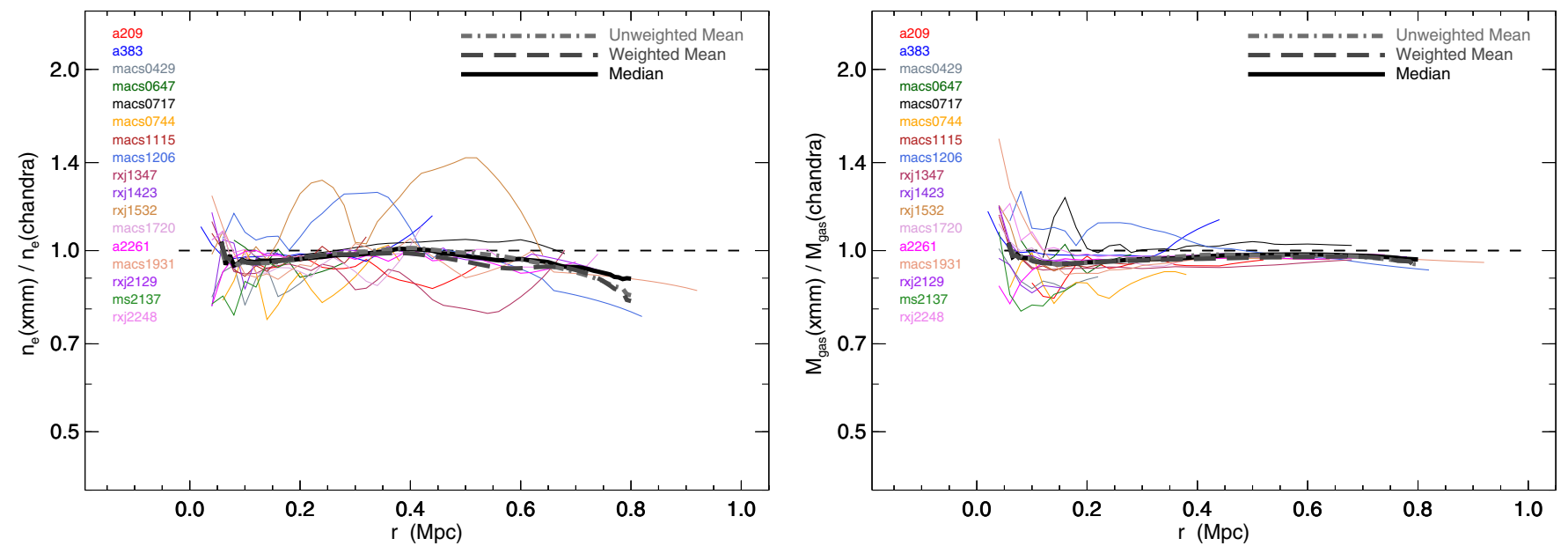

Figure 1. Average ratios of electron density $n_{e}$ (left panel) and enclosed gas mass $M_{\mathrm{gas}}$ (right panel) derived from XMM and Chandra data using JACO under the assumption of spherical symmetry. Thick solid lines show the median ratios. Long-dashed thick lines show weighted means. Dot-dashed thick lines show unweighted means. Short-dashed lines indicate the locus of equality. Lists at the left show the clusters represented, whose best-fit profile ratios are given by the thin lines.

(A color version of this figure is available in the online journal.)

Table 3

X-Ray Fit Statistics

\begin{tabular}{|c|c|c|c|c|c|c|c|c|c|c|c|c|c|c|}
\hline \multirow[t]{2}{*}{ Name } & \multicolumn{7}{|c|}{ Chandra } & \multicolumn{7}{|c|}{$X M M$} \\
\hline & $N_{\text {Rad }}$ & $N_{\mathrm{Sp}}$ & $\begin{array}{c}R_{\max } \\
(\operatorname{arcmin})\end{array}$ & dof & $\chi_{v}^{2}$ & Prob & $\begin{array}{l}S / T \\
\text { ACIS }\end{array}$ & $N_{\text {Rad }}$ & $N_{\mathrm{Sp}}$ & $\begin{array}{c}R_{\max } \\
(\operatorname{arcmin})\end{array}$ & dof & $\chi_{v}^{2}$ & Prob & $\begin{array}{c}S / T \\
\mathrm{M} 1 / \mathrm{M} 2 / \mathrm{pn}\end{array}$ \\
\hline A209 & 4 & 8 & 3.2 & 616 & 0.973 & 0.673 & 0.80 & 12 & 36 & 7.5 & 1289 & 0.937 & 0.945 & $0.3 / 0.41 / 0.27$ \\
\hline A383 & 13 & 26 & 2.7 & 1430 & 1.09 & $7 \mathrm{E}-3$ & 0.4 & 12 & 36 & 4.17 & 1553 & 1.075 & 0.019 & $0.35 / 0.31 / 0.44$ \\
\hline MACS 0329 & 5 & 10 & 1.08 & 550 & 1.22 & $3 \mathrm{E}-4$ & 0.9 & $\ldots$ & $\ldots$ & $\ldots$ & $\ldots$ & $\ldots$ & $\ldots$ & \\
\hline MACS 0429 & 5 & 5 & 0.9 & 230 & 0.98 & 0.57 & 0.93 & 8 & 24 & 4.17 & 3783 & 0.68 & 1.00 & $0.36 / 0.48 / 0.28$ \\
\hline MACS 0744 & 5 & 15 & 2.05 & 633 & 1.06 & 0.13 & 0.5 & 4 & 24 & 1.17 & 783 & 1.09 & 0.04 & $0.72 / 0.79 / 0.68$ \\
\hline A611 & 16 & 16 & 3.38 & 1024 & 0.99 & 0.57 & 0.38 & $\ldots$ & $\ldots$ & $\ldots$ & $\ldots$ & $\ldots$ & $\ldots$ & \\
\hline MACS 1115 & 10 & 10 & 1.25 & 707 & 1.01 & 0.42 & 0.87 & 8 & 24 & 3.53 & 815 & 1.03 & 0.26 & $0.54 / 0.61 / 0.87$ \\
\hline A1423 & 9 & 9 & 5 & 786 & 0.975 & 0.684 & 0.29 & $\ldots$ & $\ldots$ & $\ldots$ & $\ldots$ & $\ldots$ & $\ldots$ & $\ldots$ \\
\hline A2261 & 14 & 14 & 3.8 & 1093 & 0.902 & 0.99 & 0.72 & 16 & 48 & 5.8 & 3104 & 1.046 & 0.034 & $0.26 / 0.36 / 0.28$ \\
\hline MACS 1206 & 10 & 10 & 2.7 & 524 & 0.88 & 0.97 & 0.61 & 6 & 18 & 2.68 & 1149 & 0.98 & 0.7 & $0.44 / 0.56 / 0.41$ \\
\hline CL 1226 & 3 & 6 & 0.92 & 186 & 1.02 & 0.41 & 0.84 & 3 & 15 & 5 & 504 & 0.77 & 1.00 & $\ldots$ \\
\hline MACS 1311 & 7 & 7 & 0.85 & 305 & 0.9 & 0.89 & 0.85 & $\ldots$ & $\ldots$ & $\ldots$ & $\ldots$ & $\ldots$ & $\ldots$ & $\ldots$ \\
\hline RX J1347 & 10 & 30 & 2.54 & 5462 & 1.06 & $6 \mathrm{E}-4$ & 0.7 & 12 & 36 & 3.67 & 2787 & 104 & 0.066 & $0.43 / 0.48 / 0.34$ \\
\hline RX J1423 & 1 & 11 & 0.47 & 861 & 0.958 & 0.807 & 0.95 & $\ldots$ & $\ldots$ & $\ldots$ & $\ldots$ & $\ldots$ & $\ldots$ & $\ldots$ \\
\hline RX J1532 & 22 & 22 & 2.87 & 2756 & 0.944 & 0.98 & 0.24 & 5 & 15 & 2.83 & 540 & 1.04 & 0.24 & $0.68 / 0.59 / 0.54$ \\
\hline MACS 1720 & 6 & 12 & 1.66 & 689 & 1.03 & 0.3 & 0.75 & 8 & 24 & 3.67 & 765 & 1.03 & 0.29 & $0.12 / 0.31 / 0.17$ \\
\hline MACS 1931 & 17 & 17 & 3.69 & 2689 & 1.04 & 0.09 & 0.27 & 11 & 33 & 3.89 & 2405 & 1.03 & 0.10 & $0.26 / 0.24 / 0.15$ \\
\hline RX J2129 & 16 & 16 & 4.5 & 1202 & 0.916 & 0.98 & 0.27 & 14 & 42 & 4 & 2253 & 1.002 & 0.46 & $0.49 / 0.50 / 0.32$ \\
\hline MS 2137 & 8 & 16 & 0.82 & 1303 & 0.94 & 0.94 & 0.93 & 6 & 18 & 1.58 & 1880 & 1.11 & 0.0004 & $0.83 / 0.84 / 0.73$ \\
\hline RXC J2248 & 18 & 18 & 3.04 & 1320 & 0.925 & 0.975 & 0.72 & 13 & 30 & 3.67 & 2503 & 1.04 & 0.06 & $0.56 / 0.56 / 0.45$ \\
\hline MACS 0416 & 3 & 3 & 1.39 & 117 & 1.12 & 0.18 & 0.83 & $\ldots$ & $\ldots$ & $\ldots$ & $\ldots$ & $\ldots$ & $\ldots$ & \\
\hline MACS 0647 & 3 & 6 & 0.95 & 163 & 0.94 & 0.68 & 0.92 & 5 & 15 & 1.67 & 558 & 1.00 & 0.46 & $0.58 / 0.71 / 0.52$ \\
\hline MACS 0717 & 11 & 11 & 2.2 & 829 & 1.16 & $7 \mathrm{E}-4$ & 0.79 & 7 & 63 & 2 & 3462 & 1.01 & 0.27 & $0.71 / 0.76 / 0.59$ \\
\hline MACS 1149 & 3 & 6 & 1.44 & 292 & 0.84 & 0.98 & 0.89 & $\ldots$ & $\ldots$ & $\ldots$ & $\ldots$ & $\ldots$ & $\ldots$ & $\ldots$ \\
\hline MACS 2129 & 3 & 6 & 1.58 & 197 & 1.11 & 0.14 & 0.8 & $\ldots$ & $\ldots$ & $\ldots$ & $\ldots$ & $\ldots$ & $\ldots$ & $\ldots$ \\
\hline
\end{tabular}

Notes. $N_{\mathrm{Rad}}$ is the number of radial bins; $N_{\mathrm{Sp}}$ is the total number of spectra; $R_{\max }$ is outer radius in arcminutes of the outermost bin; dof is the number of degrees of freedom (the number of spectral energy bins minus the number of fit parameters) for the full JACO parameterized fit; $\chi_{v}^{2}$ is the $\chi^{2}$ statistic divided by the dof; Prob is the probability corresponding to the best fit. $S / T$ is the ratio of the total number of source counts $S$ divided by the total number of particle and soft X-ray counts $T$ in the $0.7-8.0 \mathrm{keV}$ band in the outermost bin, with the exception of the outermost pn observation for A383, where the ratio is the total source counts to the particle background.

virtually identical, with a slight trend to smaller densities in the $X M M$ data at large radii.

The right panel of Figure 1 shows the ratios of gas mass $M_{\text {gas }}(r)$ enclosed within radius $r$ derived by JACO from the $X M M$ and Chandra data. Integration of gas density over radius smooths out the variations seen in the electron-density fits, leading to excellent agreement in the gas-mass profiles outside of $0.2 \mathrm{Mpc}$ with a dispersion of only a few percent. It is reassuring to see such consistency between the $M_{\text {gas }}(r)$ values derived from Chandra and XMM. 


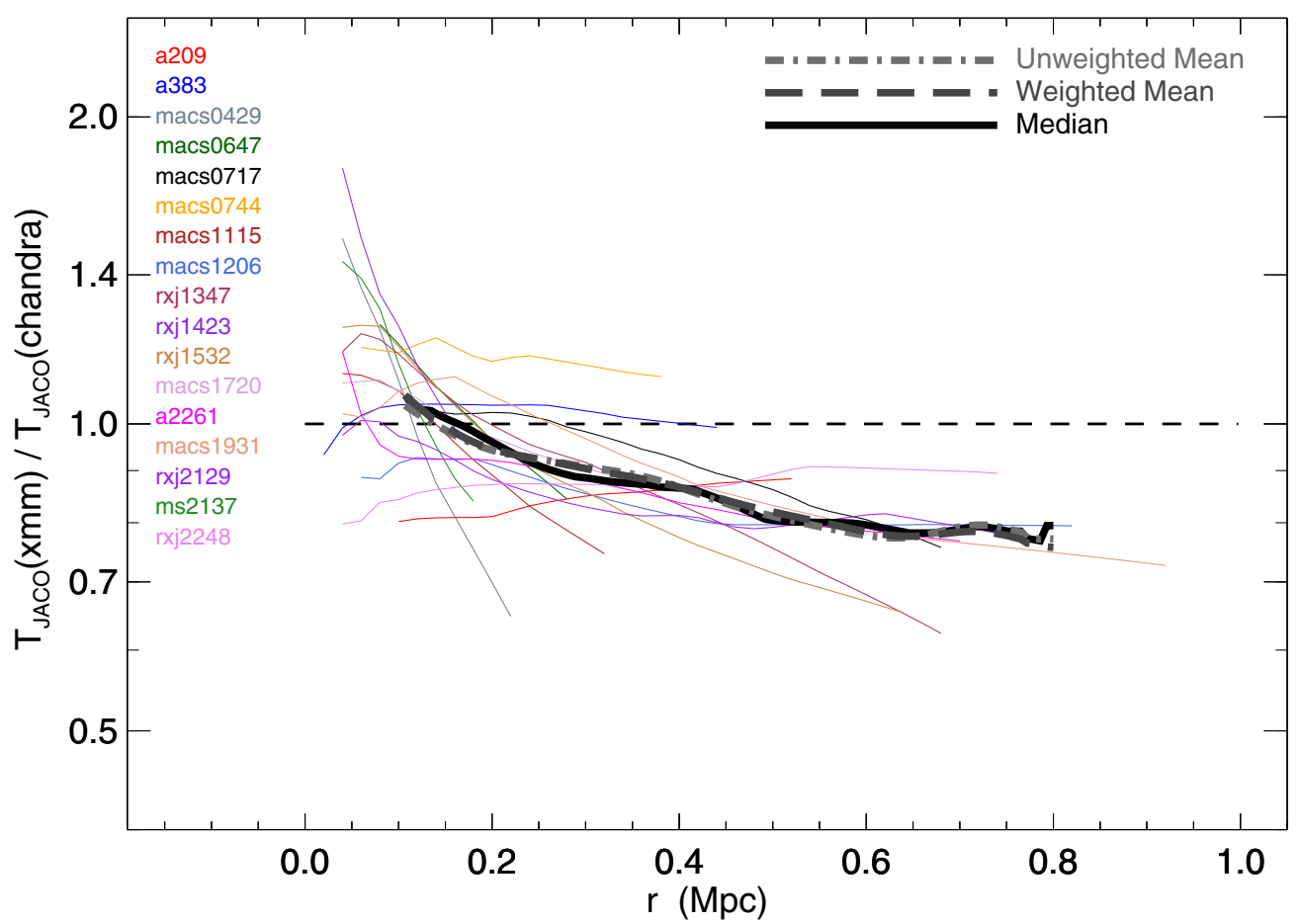

Figure 2. Average ratios of derived gas temperature $T_{\mathrm{JACO}}$ from $X M M$ and Chandra, as functions of radius. Line styles are the same as in Figure 1. (A color version of this figure is available in the online journal.)

\subsection{Temperature Profiles}

Comparisons of the temperature profiles from Chandra and $X M M$ are not as comforting. Figure 2 shows our comparisons. At $\sim 100 \mathrm{kpc}$ radii there is no systematic difference between the temperatures JACO measures from Chandra and XMM data, but the $X M M$ temperature systematically declines relative to the Chandra temperature as distance from the cluster center increases, reaching a mean ratio $\approx 0.75$ at radii approaching $1 \mathrm{Mpc}$.

Figure 3 supports this finding, because it shows that systematic temperature differences at large radii are not an artifact of the parametric JACO fitting procedure. Data points in the figure show the nonparametric JACO fits to the binned spectra, which invoke neither the assumption of HSE nor the assumption of NFW mass profiles. They simply represent the uncorrelated bin-by-bin temperatures that best fit the projected spectroscopic data. And more often than not, the XMM temperatures at $\gtrsim 0.5 \mathrm{Mpc}$ are below the Chandra temperatures, despite the fact that there is no apparent systematic temperature difference at small radii. We note, as shown in Figure 3, that the XMM temperature data for cluster CL J1226+3332 are inadequate for deriving a mass profile. We have therefore excluded this cluster from the other $X M M$ data comparisons made in this paper.

Cluster temperature discrepancies between $X M M$ and Chandra have been noticed before. For example, Mahdavi et al. (2013) found that XMM HSE cluster masses were systematically $\sim 15 \%$ smaller than Chandra HSE cluster masses if they made no attempt to correct for a systematic temperature offset. In order to bring the masses into agreement, they introduced a photon-energy dependent effective-area correction factor of $(E / \mathrm{keV})^{0.07}$, where $E$ is the photon energy, into the Chandra data analysis. An independent comparison by Schellenberger et al. (2014) shows that $X M M$ temperatures from all three detectors (MOS1, MOS2, and pn) are systematically smaller than
Chandra temperatures by a percentage that increases with cluster temperature and reaches $\sim 20 \%$ at the $\sim 8-12 \mathrm{keV}$ temperatures typical of CLASH clusters; Nevalainen et al. (2010) reach similar conclusions in a comparison of temperatures obtained from fitting to $0.5-2.0 \mathrm{keV}$ spectra compared to those obtained from fitting to $2.0-7.0 \mathrm{keV}$ spectra. In that study, the $2.0-7.0 \mathrm{keV}$ results were more similar to Chandra results than those for the $0.5-2.0 \mathrm{keV}$ bandpass.

To our knowledge, our work here shows the first indication that the temperature discrepancy depends on distance from the cluster center. The fact that our Chandra and XMM temperatures agree in the core, where photon fluxes are greatest, suggests that miscalibration of either the Chandra or the XMM effective area on-axis is unlikely to be the main problem. One example of a systematic that could produce this radial trend is excess large-angle scattering of soft X-ray photons not accounted for in the standard XMM PSF form. The regions where the temperature differences are greatest in CLASH clusters are typically $\sim 1^{\prime}$ from the much brighter cluster core. A relatively small fraction of soft photons scattered from the core into regions $\sim 1^{\prime}$ away from it could therefore produce a significant decrement in the best-fit temperature. Taking the XMM PSF into account is a challenging endeavor (e.g., Croston et al. 2008; Pointecouteau et al. 2004), and so development using the JACO analysis platform is planned for future work. Alternatively, improper background treatments for either the XMM or Chandra could result in apparent temperature differences at larger radii, but we consider that hypothesis unlikely because temperature differences persist in regions where the signal-to-background ratio is large. Finally, we cannot rule out the possibility that the $X M M$ and Chandra responses are compatible at the usual aimpoint but incur increasing discrepancies in the outer parts of the detectors. The latter possibility is not so far-fetched because most of the calibration is likely to be the best on-axis. Investigation of all of these possibilities is beyond the scope 


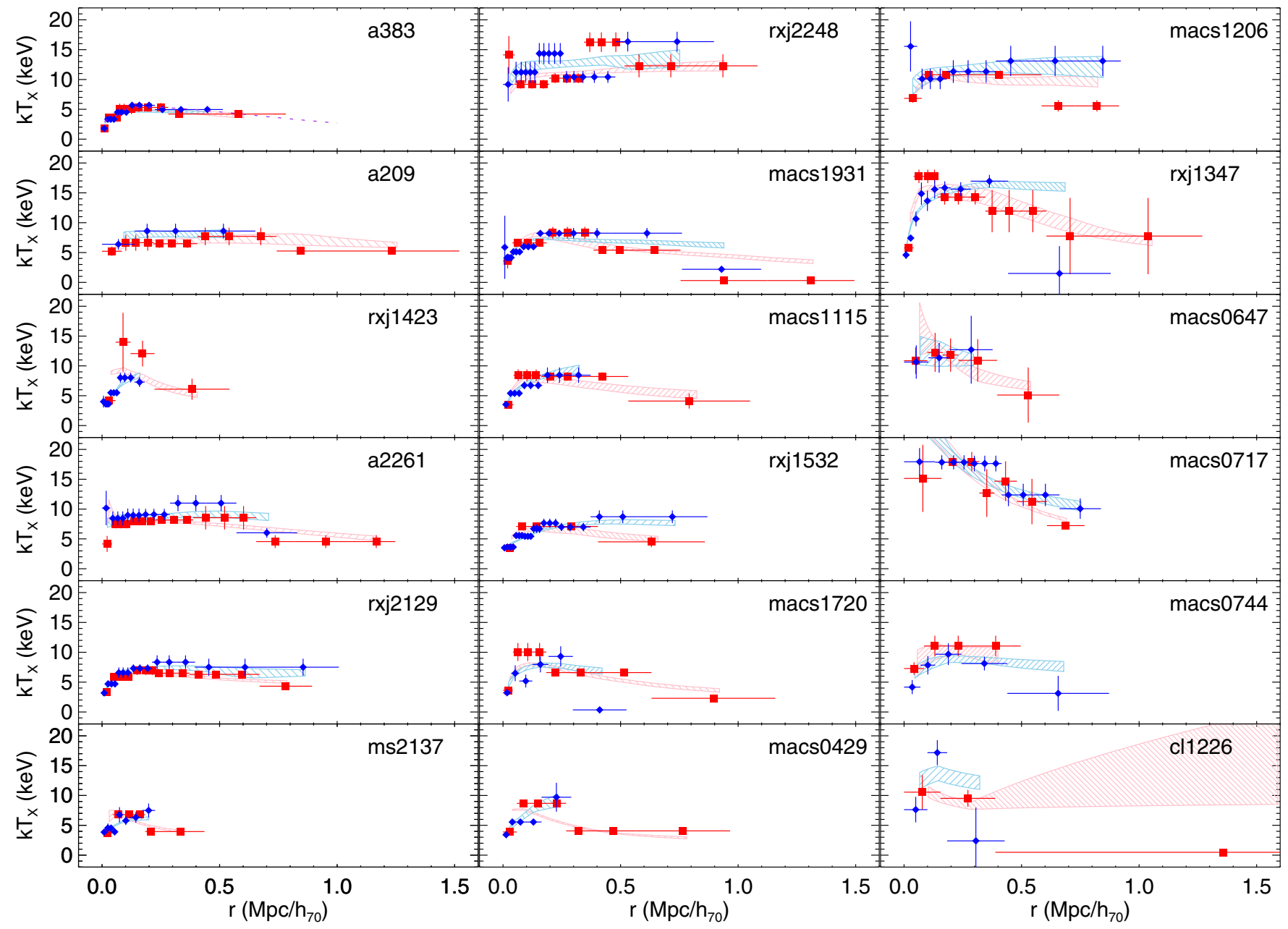

Figure 3. Temperature fits to XMM and Chandra data for CLASH clusters observed with both telescopes. Red squares show nonparametric fits to XMM data. Blue diamonds show nonparametric fits to Chandra data. Horizontal error bars on those points represent the radial ranges of the annular regions used to extract the X-ray events. Hatched areas show the temperature profiles corresponding to the best-fitting parametric JACO models, which assume hydrostatic equilibrium and NFW mass profiles. The dotted line in the A383 panel shows the best-fitting Chandra temperature profile for that cluster from Vikhlinin et al. (2006). Clusters are ordered by increasing redshift, starting from the top left and proceeding downward.

(A color version of this figure is available in the online journal.)

of this paper, but our intention is to explore these issues in a future work.

\section{MASS PROFILES}

This section presents the mass profiles derived for each CLASH cluster from all the data sets available to us at the time of publication. It presents cluster mass profiles as circular-velocity plots because in that form they are virtually independent of cosmology and therefore allow mass profiles derived assuming different cosmologies to be plotted simultaneously. The plots show that HSE mass profiles derived from Chandra observations are often nearly identical to those determined from lensing. However, the plots also show that many of the XMM mass profiles are systematically tilted to lower masses at larger radii, because of the radial dependence of the temperature offset. Sections 6 and 7 present more detailed comparisons of HSE and lensing mass profiles for Chandra and XMM, respectively.

\subsection{Circular Velocity and Enclosed Mass}

In our experience, galaxy-cluster astronomers spend altogether too much time converting masses and mass profiles back and forth among the slightly different $\Lambda \mathrm{CDM}$ cosmologies they have used to derive those masses. Much wasted effort could be avoided if they simply provided and compared mass profiles in circular-velocity form. To see why, consider this form of the HSE equation for a spherical mass configuration in which $M_{r}$ and $\theta_{r}$ represent the mass enclosed within radius $r$ and angle subtended by that radius at the cluster's distance, respectively, and $\mu m_{p}$ is the mean mass per gas particle:

$$
v_{\text {circ }}^{2}\left(\theta_{r}\right) \equiv \frac{G M_{r}}{r}=\frac{k T\left(\theta_{r}\right)}{\mu m_{p}}\left|\frac{d \ln P}{d \ln r}\right|_{\theta_{r}}
$$

Both of the factors needed to calculate $v_{\text {circ }}\left(\theta_{r}\right)$ can be derived from an X-ray observation without invoking a cosmological model, and converting to $v_{\text {circ }}(r)$ requires only an angular-size distance. Likewise, an analogous version of $v_{\text {circ }}\left(\theta_{r}\right)$ can be derived from lensing observations, for which the only cosmological dependences stem from the slightly altered distance distribution of the lensed background galaxies. (Conversion to a projected physical radius is necessary to compare mass profiles for clusters at different redshifts, but mass-bias analyses require only comparisons of mass profiles derived for the same cluster using different techniques.) 
Table 4

Chandra HSE Masses

\begin{tabular}{|c|c|c|c|c|c|c|c|c|c|c|c|c|}
\hline Name & $\begin{array}{c}v_{\max } \\
\left(\mathrm{km} \mathrm{s}^{-1}\right)\end{array}$ & $\sigma_{v_{\max }}$ & $\begin{array}{c}r_{\mathrm{s}} \\
\left(h_{70}^{-1} \mathrm{Mpc}\right)\end{array}$ & $\sigma_{r_{\mathrm{s}}}$ & $\begin{array}{c}r_{2500} \\
\left(h_{70}^{-1} \mathrm{Mpc}\right)\end{array}$ & $\sigma_{r_{2500}}$ & $\begin{array}{c}M_{2500} \\
\left(10^{14} h_{70}^{-1} M_{\odot}\right)\end{array}$ & $\sigma_{M_{2500}}$ & $\begin{array}{l}f_{\mathrm{g}, 2500} \\
\left(h_{70}^{-3 / 2}\right) \\
\end{array}$ & $\sigma_{f_{\mathrm{g}, 2500}}$ & $\begin{array}{c}r_{500} \\
\left(h_{70}^{-1} \mathrm{Mpc}\right)\end{array}$ & $\sigma_{r_{500}}$ \\
\hline A209 & 1743 & 126 & 0.745 & 0.653 & 0.47 & 0.014 & 2.49 & 0.36 & 0.11 & 0.005 & 1.31 & 0.16 \\
\hline A383 & 1184 & 29 & 0.22 & 0.017 & 0.436 & 0.007 & 1.42 & 0.07 & 0.107 & 0.003 & 0.94 & 0.021 \\
\hline MACS 0329-02 & 1485 & 80 & 0.36 & 0.10 & 0.46 & 0.017 & 2.24 & 0.24 & 0.117 & 0.007 & 1.05 & 0.056 \\
\hline MACS 0429-02 & 1496 & 171 & 0.30 & 0.10 & 0.486 & 0.034 & 2.49 & 0.57 & 0.105 & 0.01 & 1.07 & 0.105 \\
\hline MACS 0744+39 & 1631 & 84 & 0.43 & 0.16 & 0.425 & 0.015 & 2.34 & 0.24 & 0.128 & 0.007 & 1.01 & 0.07 \\
\hline A611 & 1678 & 92 & 0.57 & 0.17 & 0.552 & 0.019 & 3.20 & 0.35 & 0.088 & 0.0044 & 1.30 & 0.09 \\
\hline MACS 1115+01 & 1664 & 106 & 0.45 & 0.097 & 0.546 & 0.023 & 3.30 & 0.42 & 0.113 & 0.0077 & 1.25 & 0.091 \\
\hline A1423 & 1297 & 61 & 0.275 & 0.051 & 0.47 & 0.014 & 1.82 & 0.17 & 0.094 & 0.0037 & 1.03 & 0.042 \\
\hline MACS 1206-08 & 2002 & 148 & 0.71 & 0.507 & 0.587 & 0.03 & 4.59 & 0.68 & 0.122 & 0.0077 & 1.43 & 0.16 \\
\hline CL J1226+33* & 4975 & & 3.89 & 6.38 & 0.705 & 0.05 & 13.6 & 2.90 & 0.038 & 0.007 & 2.30 & 0.36 \\
\hline MACS $1311-03$ & 1390 & 116 & 0.32 & 0.094 & 0.42 & 0.022 & 1.80 & 0.30 & 0.108 & 0.01 & 0.95 & 0.08 \\
\hline RX J1347 & 2318 & 57 & 0.403 & 0.03 & 0.735 & 0.012 & 9.14 & 0.45 & 0.104 & 0.003 & 1.60 & 0.035 \\
\hline MACS $1423+24$ & 1579 & 146 & 0.287 & 0.047 & 0.472 & 0.026 & 2.70 & 0.50 & 0.099 & 0.0075 & 1.04 & 0.07 \\
\hline MACS $1532+30$ & 1629 & 41 & 0.46 & 0.042 & 0.525 & 0.009 & 3.00 & 0.15 & 0.119 & 0.004 & 1.22 & 0.033 \\
\hline MACS $1720+35$ & 1467 & 89 & 0.273 & 0.05 & 0.483 & 0.018 & 2.40 & 0.29 & 0.114 & 0.006 & 1.05 & 0.055 \\
\hline A2261 & 1571 & 56 & 0.304 & 0.059 & 0.567 & 0.013 & 3.24 & 0.23 & 0.1096 & 0.004 & 1.23 & 0.045 \\
\hline MACS 1931-26 & 1522 & 33 & 0.279 & 0.02 & 0.511 & 0.008 & 2.74 & 0.12 & 0.133 & 0.0035 & 1.11 & 0.023 \\
\hline RX J2129+00 & 1488 & 70 & 0.34 & 0.043 & 0.529 & 0.017 & 2.67 & 0.25 & 0.105 & 0.004 & 1.17 & 0.048 \\
\hline MS 2137-2353 & 1312 & 44 & 0.164 & 0.016 & 0.449 & 0.01 & 1.78 & 0.12 & 0.1205 & 0.0048 & 0.93 & 0.027 \\
\hline RX J2248-44 & 2272 & 125 & 0.828 & 0.326 & 0.706 & 0.025 & 7.19 & 0.79 & 0.1226 & 0.0067 & 1.71 & 0.14 \\
\hline MACS 0416-24 & 2000 & 800 & $\ldots$ & $<8$ & 0.6 & 0.1 & 3.8 & 1.4 & 0.094 & 0.016 & 2.1 & 0.35 \\
\hline MACS 0647+70 & 2600 & 640 & 1.26 & 3.58 & 0.62 & 0.07 & 6.5 & 3.2 & 0.008 & 0.013 & 1.67 & 0.55 \\
\hline MACS 0717+37 & 2300 & 110 & 0.96 & 0.38 & 0.59 & 0.02 & 5.4 & 0.5 & 0.124 & 0.004 & 1.52 & 0.13 \\
\hline MACS 1149+22 & 1700 & 200 & $\ldots$ & $<4$ & 0.49 & 0.04 & 3.1 & 0.8 & 0.123 & 0.008 & 1.10 & 0.28 \\
\hline MACS 2129-07 & 2000 & 400 & $\ldots$ & $<5$ & 0.56 & 0.06 & 4.7 & 1.7 & 0.009 & 0.015 & 1.35 & 0.62 \\
\hline
\end{tabular}

Aside from cosmological independence, circular velocities have several additional advantages over enclosed masses, and particularly over enclosed masses defined with respect to a spherical-overdensity threshold.

1. Dividing $M_{r}$ by $r$ removes the lowest-order dependence of $\ln M_{r}$ on $\ln r$, greatly relieving the compression of dynamic range along the vertical axis of an $M_{r}(r)$ plot. Systematic differences among derived mass profiles are then much easier to see by eye, because they are not so highly compressed.

2. The value of $v_{\text {circ }}(r)$ for an NFW profile is nearly constant in the vicinity of the scale radius: It reaches a maximum value $v_{\max }$ at $2.163 r_{\mathrm{s}}$, remains within $6 \%$ of $v_{\max }$ over the interval $1 \lesssim r / r_{s} \lesssim 5$, and stays within $2 \%$ of $v_{\max }$ over the interval $1.4 \lesssim r / r_{s} \lesssim 3.5$. Circular-velocity measurements are therefore much less subject to aperture-induced covariances than enclosed-mass measurements, in which correlated errors in the determinations of spherical-overdensity radii can induce systematically correlated offsets in all quantities defined with respect to those radii, complicating the task of distinguishing true observational biases from those introduced by the subsequent analysis.

3. If a dark matter halo is not changing, then there is no evolution in $r_{\mathrm{s}}$ or $v_{\max }$. The same cannot be said for $r_{\Delta}$, $M_{\Delta}$, or $c_{\Delta}$, which continue to change simply because the density threshold used to measure them declines with time. A halo's value of $v_{\max }$ is therefore a more direct indicator of its rarity than $M_{\Delta}$, because it is independent of redshift.

4. Accurate estimates of $v_{\max }$ are often easier to obtain from X-ray observations than accurate estimates of $M_{500}$ or $M_{200}$ because the $v_{\text {circ }}$ profile in an NFW potential well with concentration $c_{200} \sim 4$ peaks near $r_{2500}$. An exact value of $v_{\max }$ can be hard to measure because of the lack of curvature in the $v_{\text {circ }}(r)$ profile in the vicinity of its peak, but that same feature is advantageous for making accurate estimates, because $v_{\max }$ can be reliably estimated from any set of $r-M_{r}$ pairs measured in the $\sim 0.3-2 \mathrm{Mpc}$ range.

Because of these considerations, we provide NFW profile fits in the form of $r_{\mathrm{s}}-v_{\max }$ pairs for Chandra in Table 4 and for $X M M$ in Table 5. The NFW mass profile in terms of those quantities is

$$
M_{r}=4.625 \frac{v_{\max }^{2} r}{G}\left[\frac{\ln (1+x)}{x}-\frac{1}{1+x}\right],
$$

where $x=r / r_{\mathrm{s}}$. The same tables also provide conventional $M_{2500}, r_{2500}$, and $r_{500}$ values, along with the enclosed gas fraction $f_{\mathrm{g}, 2500}=M_{\mathrm{gas}}(r) / M_{r}$ measured at $r_{2500}$. Uncertainties in the table correspond to the $68 \%$ confidence regions from the JACO MCMC analysis.

\subsection{Mass Profiles in Circular-velocity Form}

Figure 4 shows examples of mass profiles in the form of $v_{\text {circ }}$ curves for CLASH cluster RX J2129+00. A dot-dashed (blue) line shows the mass profile derived from Chandra data by the JACO fitting procedure, assuming HSE. A shortdashed (red) line shows the analogous JACO mass profile derived from $X M M$ data. Hatched regions show $68 \%$ confidence intervals from the JACO MCMC chain. Both profiles are free of cosmological assumptions when viewed as $v_{\text {circ }}\left(\theta_{r}\right)$ curves. The bottom axis shows radial distance (in $h_{70}^{-1} \mathrm{Mpc}$ ) from the cluster center, which is uncertain by $\lesssim 1 \%$ at $z \lesssim 0.5$ within a flat $\Lambda$ CDM cosmology in which the value of $\widetilde{\Omega}_{\mathrm{M}}$ is known to $\lesssim 10 \%$. Cosmological uncertainties in the positions of the light-gray diagonal lines showing enclosed mass are therefore also $\lesssim 1 \%$. 


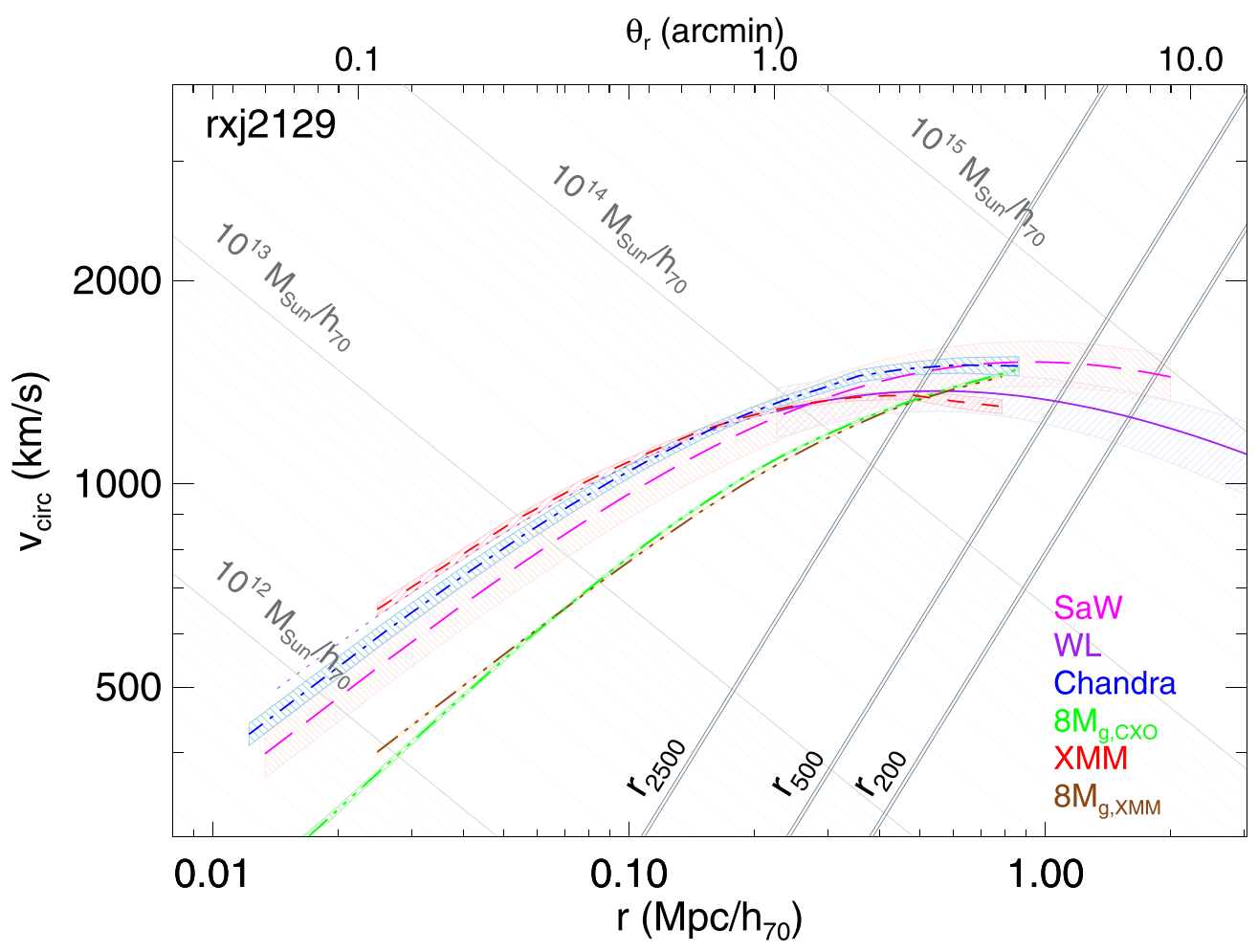

Figure 4. Observed 3D deprojected mass profiles for RX J2129, presented in $v_{\text {circ }}(r)$ form and derived from the SaWLens (long-dashed line), WL (solid line), Chandra/JACO (dot-dashed line), and XMM/JACO (short-dashed line) data sets. Also shown are gas-mass profiles, multiplied by a factor of eight, from the parametric JACO analyses of Chandra (light triple dot-dashed line) and XMM (dark triple dot-dashed line) data. In each case, hatched areas show the 68\% confidence regions. The hatched area for the weak-lensing (WL) data does not extend inside of $1^{\prime}$, because the WL fit is less reliable there, but a dotted line shows the inward extrapolation of that fit.

(A color version of this figure is available in the online journal.)

Table 5

$X M M$ HSE Masses and Maximum Circular Velocities

\begin{tabular}{|c|c|c|c|c|c|c|c|c|c|c|c|c|}
\hline Name & $\begin{array}{c}v_{\max } \\
\left(\mathrm{km} \mathrm{s}^{-1}\right)\end{array}$ & $\sigma_{v_{\max }}$ & $\begin{array}{c}r_{\mathrm{s}} \\
\left(h_{70}^{-1} \mathrm{Mpc}\right)\end{array}$ & $\sigma_{r_{\mathrm{s}}}$ & $\begin{array}{c}r_{2500} \\
\left(h_{70}^{-1} \mathrm{Mpc}\right)\end{array}$ & $\sigma_{r_{2500}}$ & $\begin{array}{c}M_{2500} \\
\left(10^{14} h_{70}^{-1} M_{\odot}\right)\end{array}$ & $\sigma_{M_{2500}}$ & $\begin{array}{l}f_{\mathrm{g}, 2500} \\
\left(h_{70}^{-3 / 2}\right) \\
\end{array}$ & $\sigma_{f_{\mathrm{g}, 2500}}$ & $\begin{array}{c}r_{500} \\
\left(h_{70}^{-1} \mathrm{Mpc}\right) \\
\end{array}$ & $\sigma_{r_{500}}$ \\
\hline A209 & 1506 & 48 & 0.81 & 0.17 & 0.46 & 0.01 & 1.73 & 0.11 & 0.125 & 0.003 & 1.2 & 0.03 \\
\hline A383 & 1243 & 27 & 0.24 & 0.02 & 0.45 & 0.007 & 1.61 & 0.07 & 0.095 & 0.003 & 0.98 & 0.02 \\
\hline MACS $0429-02$ & 1347 & 25 & 0.059 & 0.003 & 0.4 & 0.004 & 1.36 & 0.05 & 0.135 & 0.002 & 0.77 & 0.01 \\
\hline MACS $0744+39$ & 1568 & 65 & 0.14 & 0.02 & 0.43 & 0.013 & 2.4 & 0.2 & 0.115 & 0.005 & 0.88 & 0.03 \\
\hline MACS $1115+01$ & 1546 & 51 & 0.25 & 0.03 & 0.52 & 0.011 & 2.89 & 0.19 & 0.12 & 0.005 & 1.1 & 0.03 \\
\hline MACS $1206-08$ & 1974 & 70 & 0.64 & 0.15 & 0.59 & 0.013 & 4.66 & 0.32 & 0.12 & 0.006 & 1.4 & 0.1 \\
\hline CL J1226+33* & 3667 & 220 & 0.2 & 0.2 & 0.87 & 0.03 & 25 & 3 & 0.095 & 0.003 & 3.7 & 0.3 \\
\hline RX J1347 & 2096 & 105 & 0.19 & 0.02 & 0.65 & 0.022 & 6.4 & 0.64 & 0.122 & 0.005 & 1.33 & 0.05 \\
\hline MACS $1423+24$ & 1534 & 68 & 0.06 & 0.01 & 0.413 & 0.012 & 1.8 & 0.2 & 0.11 & 0.01 & 0.80 & 0.03 \\
\hline MACS $1532+30$ & 1423 & 61 & 0.18 & 0.03 & 0.475 & 0.014 & 2.22 & 0.19 & 0.138 & 0.008 & 1.00 & 0.04 \\
\hline MACS $1720+35$ & 1409 & 37 & 0.16 & 0.02 & 0.461 & 0.008 & 2.1 & 0.11 & 0.118 & 0.003 & 0.95 & 0.02 \\
\hline A2261 & 1491 & 24 & 0.28 & 0.03 & 0.539 & 0.006 & 2.78 & 0.09 & 0.116 & 0.002 & 1.15 & 0.03 \\
\hline MACS $1931-26$ & 1489 & 35 & 0.23 & 0.03 & 0.502 & 0.007 & 2.59 & 0.12 & 0.133 & 0.003 & 1.07 & 0.03 \\
\hline RX J2129+00 & 1331 & 30 & 0.21 & 0.02 & 0.478 & 0.008 & 1.97 & 0.09 & 0.122 & 0.003 & 1.01 & 0.02 \\
\hline MS 2137-2353 & 1297 & 36 & 0.06 & 0.01 & 0.401 & 0.007 & 1.27 & 0.07 & 0.133 & 0.004 & 0.78 & 0.02 \\
\hline RX J2248-44 & 2265 & 54 & 1.02 & 0.18 & 0.668 & 0.011 & 6.08 & 0.29 & 0.133 & 0.004 & 1.70 & 0.07 \\
\hline MACS $0647+70$ & 1753 & 144 & 0.22 & 0.09 & 0.52 & 0.03 & 3.7 & 0.6 & 0.105 & 0.007 & 1.1 & 0.1 \\
\hline MACS $0717+37$ & 2065 & 40 & 0.6 & 0.15 & 0.59 & 0.007 & 5.2 & 0.2 & 0.129 & 0.002 & 1.4 & 0.05 \\
\hline
\end{tabular}

Another set of darker gray diagonal lines shows the loci of the spherical-overdensity radii $r_{2500}, r_{500}$, and $r_{200}$. Two lines for each $r_{\Delta}$ indicate the cosmological uncertainty corresponding to $\Omega_{\mathrm{M}}=0.3 \pm 0.03$ at the redshift of the cluster. Intersections between these lines and the $v_{\text {circ }}(r)$ curves give the values of $M_{\Delta}$ determined by each method. Notice that systematic differences in $M_{\Delta}$, measured along lines of constant $r_{\Delta}$, are larger than the systematic differences in $M_{r}$, measured in the vertical direction, by an amount that depends on the local slopes of the $v_{\text {circ }}(r)$ curves. Measurements of the average mass-bias factor $\left\langle b_{X}\right\rangle$ therefore depend on whether it is measured at fixed physical radius or at fixed overdensity. This is one manifestation of the effect sometimes called aperture-induced covariance, in which uncertainties in mass measurement techniques produce 
correlated uncertainties in all quantities measured with respect to a spherical-overdensity radius.

Solid (purple) and long-dashed (magenta) lines show the mass profiles inferred from the CLASH WL and combined strongand-weak lensing data. The WL profile at angular radii $<1^{\prime}$, where the WL fit becomes less statistically secure, is shown with a dotted line. There is an additional systematic uncertainty of $\pm 8 \%$ in the overall mass calibration of CLASH WL (Umetsu et al. 2014). Such a systematic would serve to move all profiles in the analysis up or down. When shown as $v_{\text {circ }}(\theta)$ curves, the only sensitivity to cosmological assumptions in these lensing mass profiles comes from slight shifts in the distance distribution of the lensed background galaxies.

Figure 4 also shows radial gas-mass profiles inferred by JACO from Chandra (light green triple-dot-dashed line) and XMM (dark brown triple-dot-dashed line). We have multiplied these profiles by a factor of eight, because the ratio of gas mass to total mass typically found at $\gtrsim r_{2500}$ in massive relaxed clusters is $\approx 1 / 8$ (Vikhlinin et al. 2003; Allen et al. 2004, 2002; Mantz et al. 2008; Ettori et al. 2009). We therefore expect $8 M_{\text {gas }}$ to be a reasonably accurate mass-profile estimator outside the cores of clusters and can test this expectation with the CLASH cluster observations (see Section 8).

Figures 5 and 6 provide mass profiles in $v_{\text {circ }}(r)$ form for the other 24 CLASH clusters, in which several patterns can be seen.

1. Chandra HSE profiles generally have shapes similar to the lensing-mass profiles, with offsets to both greater and lesser masses.

2. XMM HSE profiles tend to be tilted to higher masses at small radii and lower masses at larger radii, compared to the Chandra HSE and lensing profiles. This tilt is a direct consequence of the temperature trend shown in Figure 2.

3. The $8 M_{\text {gas }}$ profiles from both Chandra and $X M M$ are quite consistent with the other mass measures at $\gtrsim r_{2500}$, even in the dramatically unrelaxed high-magnification subset. Therefore, gas mass appears to be a robust mass proxy.

4. X-ray HSE masses for the unrelaxed, high-magnification subset have larger uncertainties than in the more centrally concentrated clusters but are reasonably similar to the WL masses in radial regions where the mass profiles overlap.

The following three sections focus more closely on comparisons of Chandra HSE mass, XMM HSE mass, and $M_{\text {gas }}$ with the lensing-mass profiles presented in Umetsu et al. (2014) and Merten et al. (2014).

\section{CHANDRA-LENSING COMPARISON}

The CLASH cluster sample does not provide a definitive calibration of the ratio of Chandra HSE mass to lensing mass, because it is not a statistically complete sample, defined with respect to a particular survey threshold, such as an X-ray flux limit, SZ signal-to-noise ratio, or optical richness. It is also rather small for the purpose, currently containing only 18 clusters with mass profiles from both Chandra and Subaru WL data. And even among that set, only 11 have regions of significant radial overlap. Also, we note that by requiring the mass profiles to fit an NFW profile, we are not yet accounting for the effect on the gravitational potential of the brightest cluster galaxy (BCG), so interpretation of X-ray and SaWLens results inside 50-100 kpc should be made with caution. On the other hand, the overall quality and large radial range of the CLASH lensing data make it an excellent sample for identifying systematic differences between these mass-profile measurement techniques.
Figure 7 shows the ratios of Chandra HSE mass to lensing mass we obtain from the CLASH clusters as a function of physical radius. The left panel compares Chandra with lensingmass profiles from Subaru WL alone. The right panel compares Chandra with lensing-mass profiles from the SaWLens combination of Subaru WL data with Hubble data. For clarity we suppress the uncertainty ranges for individual clusters, which can be inferred from the plots in Section 5. Dotted lines show extensions of the WL mass-profile fits within $1^{\prime}$, where the fits become less reliable.

In the left panel, there is no significant radial trend in the massprofile ratios, indicating that one should expect similar NFW scale radii and concentrations from both mass-measurement methods. The scatter is quite significant, with a standard deviation of 0.07 about the unweighted mean at $0.5 \mathrm{Mpc}$, but this is within the range expected from projected large-scale structure in the WL measurements (e.g., Becker \& Kravtsov 2011).

The right panel shows no strong radial trend at $\gtrsim 0.2 \mathrm{Mpc}$ in the comparison to SaWLens profiles, but there is a systematic mass excess in the Chandra profiles within that radius, rising to $\sim 20 \%$ inside $\sim 100 \mathrm{kpc}$. An astrophysical origin for this excess is implausible, since it would imply that the potential well at small radii is insufficient for balancing the pressure of the hot gas at small radii. One potential algorithmic origin is inaccuracy of the NFW mass model used to do the fitting, which does not account for the distribution of the stellar mass of the massive galaxies at the centers of many of these clusters. As one moves inward from $\sim 100 \mathrm{kpc}$, the stellar mass fraction becomes increasingly important, and it dominates at $\lesssim 10 \mathrm{kpc}$.

Both strong-lensing and X-ray HSE techniques are sensitive to the stellar mass of the BCG, but stellar mass is likely to have a greater effect on the best-fitting X-ray mass profile in a relaxed galaxy cluster because the prominence of the central $\mathrm{X}$-ray surface-brightness peak causes the innermost parts of an $\mathrm{X}$-ray observation to have greater statistical weight. This effect will be greatest in clusters with the sharpest central peaks in $\mathrm{X}$-ray brightness, which are the ones with the lowest values of core entropy $\left(K_{0}\right)$. One can see signs of the effect in Figures 5 and 6. Virtually all of the clusters in which the Chandra HSE mass significantly exceeds the SaWLens mass belong to the low-entropy subset in Figure 5, with the greatest differences in RX J1347, MACS 1931, MS 2137, MACS 1115, RX J1532, and MACS 0429, all of which have $K_{0}<20 \mathrm{keV} \mathrm{cm}^{2}$. Among the higher core-entropy subset in Figure 6, only the lowestentropy example shows such a Chandra mass excess at small radii: A2261 $\left(K_{0}=61.1 \mathrm{keV} \mathrm{cm}^{2}\right)$.

Table 6 gives the average mass biases we find in CLASH-X in terms of mass-ratio factors $\langle f\rangle \equiv 1-\langle b\rangle$. We have chosen to compute Chandra-lensing mass bias at $0.5 \mathrm{Mpc}$ because a comparison at that radius includes almost all of the CLASH clusters with significant regions of radial overlap between our Chandra and WL mass profiles. It is also in the vicinity of $r_{2500}$ for all of these clusters. We have chosen not to compare mass biases at $r_{2500}$ in order to avoid introducing additional systematic effects stemming from uncertainties in the mass measurements from which $r_{2500}$ is inferred. The table lists both a weighted and an unweighted mean mass-ratio factor, $\langle f\rangle$, which is a geometric mean computed at radius $r$, along with the uncertainty $\sigma_{\langle f\rangle}$ in each mean, the fractional standard deviation $\sigma_{\ln f}$ about the mean, a reduced $\chi_{v}^{2}$ value based on the formal uncertainties in the cluster mass measurements, and the intrinsic fractional dispersion $\sigma_{\ln f}^{\text {intrinsic }}$ that remains, in order to obtain a reduced 


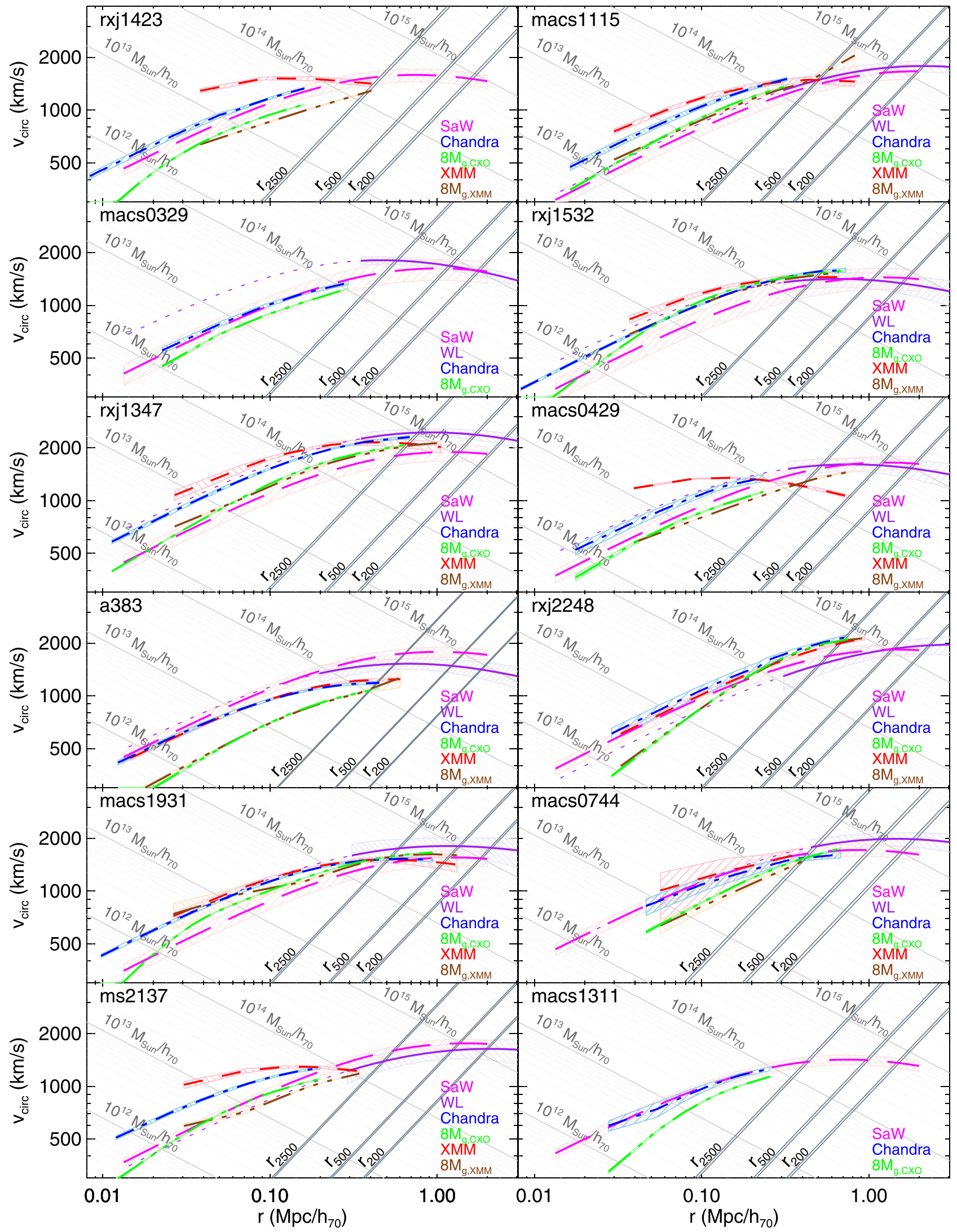

Figure 5. Circular velocity plots for the 12 CLASH clusters with $K_{0}<50 \mathrm{keV} \mathrm{cm}^{2}$. Line coding and conventions are the same as in Figure 4 . Core entropy ( $K_{0}$ ) increases from $10.2 \mathrm{keV} \mathrm{cm}^{2}$ to $14.7 \mathrm{keV} \mathrm{cm}^{2}$ proceeding down the left column and from $14.8 \mathrm{keV} \mathrm{cm}^{2}$ to $47.4 \mathrm{keV} \mathrm{cm}{ }^{2}$ proceeding down the right column.

(A color version of this figure is available in the online journal.) 


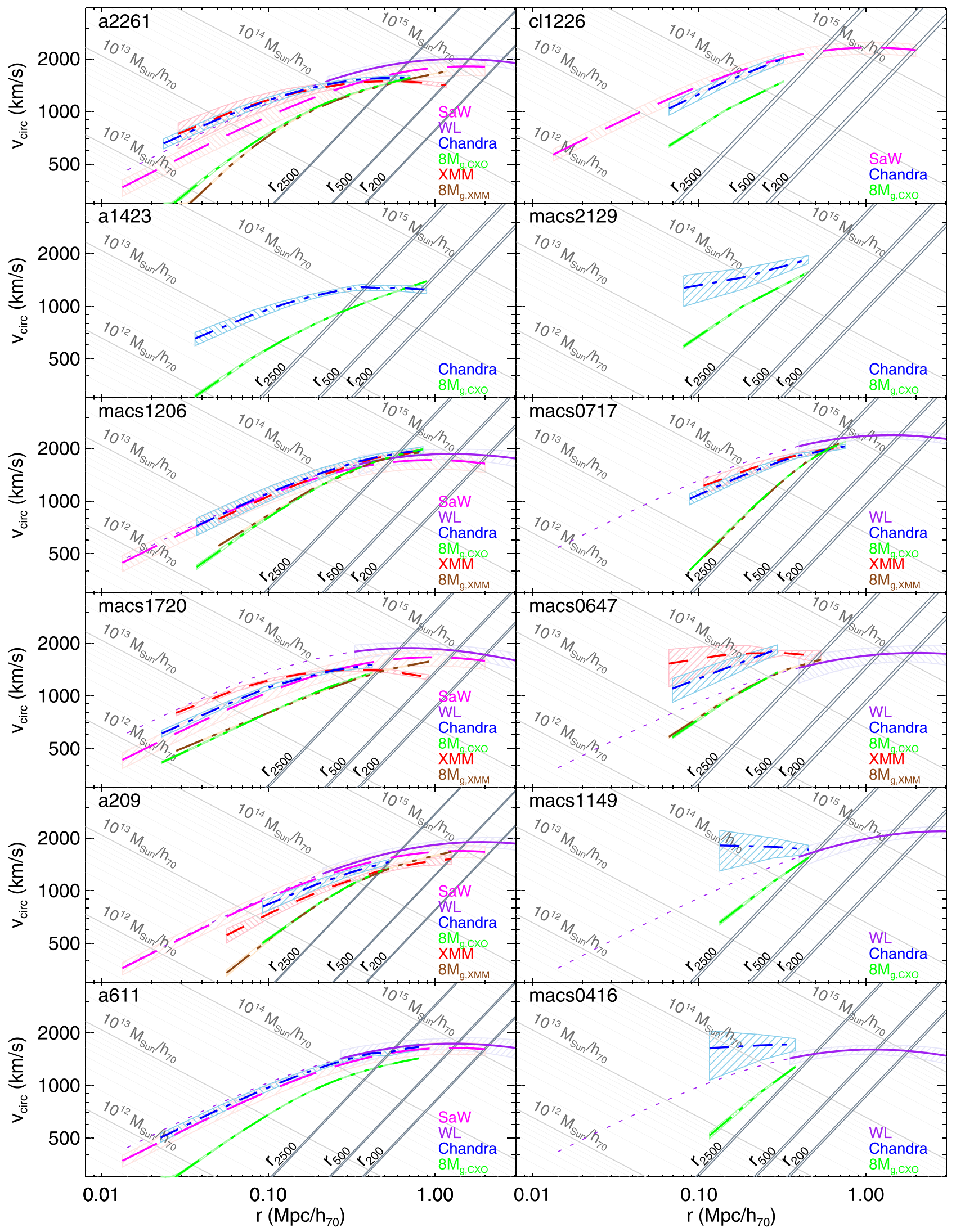

Figure 6. Circular velocity plots for the $12 \mathrm{CLASH}$ clusters with $K_{0}>50 \mathrm{keV} \mathrm{cm}^{2}$. Line coding and conventions are the same as in Figure 4 . Core entropy ( $K_{0}$ ) increases from $61.1 \mathrm{keV} \mathrm{cm}^{2}$ to $125 \mathrm{keV} \mathrm{cm}^{2}$ proceeding down the left column and from $166 \mathrm{keV} \mathrm{cm}^{2}$ to $400 \mathrm{keV} \mathrm{cm}^{2}$ proceeding down the right column. The last five clusters in the right column are the high-magnification subset.

(A color version of this figure is available in the online journal.) 
Table 6

Average Mass Bias in Terms of Mass Ratio: $f=1-b=M_{1} / M_{2}$

\begin{tabular}{|c|c|c|c|c|c|c|c|c|c|}
\hline$M_{1}$ & $M_{2}$ & Weighting & $\begin{array}{c}r \\
\left(h_{70}^{-1} \mathrm{Mpc}\right)\end{array}$ & $N_{\mathrm{cl}}$ & $\langle f\rangle$ & $\sigma_{\langle f\rangle}$ & $\sigma_{\ln f}$ & $\chi_{v}^{2}$ & $\sigma_{\ln f}^{\text {intrinsic }}$ \\
\hline$M_{\text {chandra }}$ & $M_{\mathrm{wl}}$ & Unweighted & 0.5 & 11 & 0.95 & 0.07 & 0.27 & 2.52 & 0.19 \\
\hline$M_{\text {chandra }}$ & $M_{\mathrm{wl}}$ & Weighted & 0.5 & 11 & 0.88 & 0.07 & 0.22 & 2.22 & 0.16 \\
\hline$M_{\text {chandra }}$ & $M_{\text {SaWLens }}$ & Unweighted & 0.5 & 10 & 1.13 & 0.07 & 0.20 & 1.17 & 0.08 \\
\hline$M_{\text {chandra }}$ & $M_{\text {SaWLens }}$ & Weighted & 0.5 & 10 & 1.11 & 0.07 & 0.19 & 1.16 & 0.07 \\
\hline$M_{\mathrm{xmm}}$ & $M_{\mathrm{wl}}$ & Unweighted & 0.5 & 14 & 0.84 & 0.06 & 0.30 & 2.91 & 0.21 \\
\hline$M_{\mathrm{xmm}}$ & $M_{\mathrm{wl}}$ & Weighted & 0.5 & 14 & 0.76 & 0.05 & 0.23 & 2.46 & 0.18 \\
\hline$M_{\mathrm{xmm}}$ & $M_{\text {SaWLens }}$ & Unweighted & 0.5 & 13 & 0.89 & 0.10 & 0.33 & 3.64 & 0.30 \\
\hline$M_{\mathrm{xmm}}$ & $M_{\text {SaWLens }}$ & Weighted & 0.5 & 13 & 0.82 & 0.08 & 0.33 & 3.45 & 0.28 \\
\hline$M_{\mathrm{xmm}}$ & $M_{\mathrm{wl}}$ & Unweighted & 0.8 & 8 & 0.77 & 0.09 & 0.36 & 5.93 & 0.32 \\
\hline$M_{\mathrm{xmm}}$ & $M_{\mathrm{wl}}$ & Weighted & 0.8 & 8 & 0.69 & 0.08 & 0.30 & 5.28 & 0.28 \\
\hline$M_{\mathrm{xmm}}$ & $M_{\text {SaWLens }}$ & Unweighted & 0.8 & 8 & 0.95 & 0.10 & 0.29 & 2.15 & 0.22 \\
\hline$M_{\mathrm{xmm}}$ & $M_{\text {SaWLens }}$ & Weighted & 0.8 & 8 & 0.96 & 0.11 & 0.28 & 2.14 & 0.21 \\
\hline$M_{\mathrm{xmm}}$ & $M_{\mathrm{wl}}$ & Unweighted & 1.1 & 3 & 0.60 & 0.09 & 0.13 & 1.30 & 0.08 \\
\hline$M_{\mathrm{xmm}}$ & $M_{\mathrm{wl}}$ & Weighted & 1.1 & 3 & 0.56 & 0.05 & 0.12 & 0.99 & $\ldots$ \\
\hline$M_{\mathrm{xmm}}$ & $M_{\text {SaWLens }}$ & Unweighted & 1.1 & 3 & 0.76 & 0.07 & 0.16 & 0.52 & $\ldots$ \\
\hline$M_{\mathrm{xmm}}$ & $M_{\text {SaWLens }}$ & Weighted & 1.1 & 3 & 0.75 & 0.07 & 0.10 & 0.52 & $\ldots$ \\
\hline$M_{\text {chandra }}$ & $M_{\mathrm{wl}}$ & Unweighted & $r_{500}$ & 20 & 0.91 & 0.12 & 0.76 & 3.83 & 0.50 \\
\hline$M_{\text {chandra }}$ & $M_{\mathrm{wl}}$ & Weighted & $r_{500}$ & 20 & 0.78 & 0.10 & 0.54 & 3.53 & 0.46 \\
\hline$M_{\text {chandra }}$ & $M_{\text {SaWLens }}$ & Unweighted & $r_{500}$ & 19 & 0.95 & 0.15 & 0.66 & 11.3 & 0.65 \\
\hline$M_{\text {chandra }}$ & $M_{\text {SaWLens }}$ & Weighted & $r_{500}$ & 19 & 0.69 & 0.09 & 0.58 & 8.7 & 0.54 \\
\hline$M_{\mathrm{xmm}}$ & $M_{\mathrm{wl}}$ & Unweighted & $r_{500}$ & 16 & 0.59 & 0.07 & 0.52 & 3.44 & 0.38 \\
\hline$M_{\mathrm{xmm}}$ & $M_{\mathrm{wl}}$ & Weighted & $r_{500}$ & 16 & 0.56 & 0.06 & 0.43 & 3.39 & 0.36 \\
\hline$M_{\mathrm{xmm}}$ & $M_{\text {SaWLens }}$ & Unweighted & $r_{500}$ & 15 & 0.61 & 0.10 & 0.61 & 15.7 & 0.60 \\
\hline$M_{\mathrm{xmm}}$ & $M_{\text {SaWLens }}$ & Weighted & $r_{500}$ & 15 & 0.53 & 0.08 & 0.58 & 14.7 & 0.56 \\
\hline$M_{\text {SaWLens }}$ & $M_{\mathrm{wl}}$ & Unweighted & 0.5 & 16 & 0.89 & 0.05 & 0.23 & 0.90 & $\ldots$ \\
\hline$M_{\text {SaWLens }}$ & $M_{\mathrm{wl}}$ & Weighted & 0.5 & 16 & 0.91 & 0.05 & 0.23 & 0.89 & $\ldots$ \\
\hline$M_{\text {SaWLens }}$ & $M_{\mathrm{wl}}$ & Unweighted & 1.0 & 16 & 0.92 & 0.05 & 0.22 & 1.00 & $\ldots$ \\
\hline$M_{\text {SaWLens }}$ & $M_{\mathrm{wl}}$ & Weighted & 1.0 & 16 & 0.93 & 0.05 & 0.23 & 1.00 & $\ldots$ \\
\hline$M_{\text {SaWLens }}$ & $M_{\mathrm{wl}}$ & Unweighted & $r_{500}$ & 16 & 0.90 & 0.06 & 0.29 & 1.12 & 0.09 \\
\hline$M_{\text {SaWLens }}$ & $M_{\mathrm{wl}}$ & Weighted & $r_{500}$ & 16 & 0.89 & 0.07 & 0.27 & 1.12 & 0.09 \\
\hline$M_{\text {gas }}($ Chandra $)$ & $M_{\mathrm{wl}}$ & Unweighted & 0.5 & 11 & 0.107 & 0.008 & 0.30 & 4.01 & 0.24 \\
\hline$M_{\mathrm{gas}}($ Chandra $)$ & $M_{\mathrm{wl}}$ & Weighted & 0.5 & 11 & 0.094 & 0.007 & 0.23 & 2.98 & 0.19 \\
\hline$M_{\text {gas }}($ Chandra $)$ & $M_{\text {SaWLens }}$ & Unweighted & 0.5 & 10 & 0.128 & 0.010 & 0.24 & 1.90 & 0.17 \\
\hline$M_{\text {gas }}($ Chandra $)$ & $M_{\text {SaWLens }}$ & Weighted & 0.5 & 10 & 0.124 & 0.010 & 0.23 & 1.85 & 0.16 \\
\hline$M_{\text {gas }}(X M M)$ & $M_{\mathrm{wl}}$ & Unweighted & 0.5 & 14 & 0.106 & 0.008 & 0.30 & 3.80 & 0.25 \\
\hline$M_{\text {gas }}(X M M)$ & $M_{\mathrm{wl}}$ & Weighted & 0.5 & 15 & 0.093 & 0.006 & 0.25 & 2.95 & 0.21 \\
\hline$M_{\mathrm{gas}}(X M M)$ & $M_{\text {SaWLens }}$ & Unweighted & 0.5 & 12 & 0.120 & 0.010 & 0.31 & 2.71 & 0.25 \\
\hline$M_{\text {gas }}(X M M)$ & $M_{\text {SaWLens }}$ & Weighted & 0.5 & 12 & 0.117 & 0.011 & 0.35 & 2.70 & 0.24 \\
\hline$M_{\text {gas }}($ Chandra $)$ & $M_{\mathrm{wl}}$ & Unweighted & 0.8 & 4 & 0.118 & 0.015 & 0.25 & 2.75 & 0.21 \\
\hline$M_{\text {gas }}($ Chandra $)$ & $M_{\mathrm{wl}}$ & Weighted & 0.8 & 4 & 0.121 & 0.016 & 0.23 & 2.71 & 0.18 \\
\hline$M_{\text {gas }}($ Chandra $)$ & $M_{\text {SaWLens }}$ & Unweighted & 0.8 & 4 & 0.128 & 0.014 & 0.23 & 1.71 & 0.15 \\
\hline$M_{\text {gas }}($ Chandra $)$ & $M_{\text {SaWLens }}$ & Weighted & 0.8 & 4 & 0.122 & 0.013 & 0.19 & 1.60 & 0.12 \\
\hline$M_{\text {gas }}(X M M)$ & $M_{\mathrm{wl}}$ & Unweighted & 0.8 & 8 & 0.114 & 0.013 & 0.36 & 6.59 & 0.29 \\
\hline$M_{\mathrm{gas}}(X M M)$ & $M_{\mathrm{wl}}$ & Weighted & 0.8 & 8 & 0.095 & 0.008 & 0.23 & 3.97 & 0.20 \\
\hline$M_{\mathrm{gas}}(X M M)$ & $M_{\text {SaWLens }}$ & Unweighted & 0.8 & 8 & 0.141 & 0.012 & 0.27 & 1.45 & 0.13 \\
\hline$M_{\mathrm{gas}}(X M M)$ & $M_{\text {SaWLens }}$ & Weighted & 0.8 & 8 & 0.135 & 0.011 & 0.22 & 1.41 & 0.12 \\
\hline$M_{\mathrm{gas}}(X M M)$ & $M_{\mathrm{wl}}$ & Unweighted & 1.1 & 3 & 0.095 & 0.003 & 0.06 & 0.32 & $\ldots$ \\
\hline$M_{\mathrm{gas}}(X M M)$ & $M_{\mathrm{wl}}$ & Weighted & 1.1 & 3 & 0.093 & 0.010 & 0.22 & 1.43 & 0.12 \\
\hline$M_{\mathrm{gas}}(X M M)$ & $M_{\text {SaWLens }}$ & Unweighted & 1.1 & 3 & 0.120 & 0.007 & 0.11 & 0.28 & $\ldots$ \\
\hline$M_{\mathrm{gas}}(X M M)$ & $M_{\text {SaWLens }}$ & Weighted & 1.1 & 3 & 0.121 & 0.007 & 0.08 & 0.28 & $\ldots$ \\
\hline
\end{tabular}

Note. There is an additional systematic uncertainty of $8 \%$ in the overall mass calibration for the weak-lensing profiles for CLASH.

chi-squared of unity after accounting for the formal statistical uncertainties.

Values of the mass-ratio factors for both the Chandra-WL and Chandra-SaWLens comparisons at $0.5 \mathrm{Mpc}$ are within $15 \%$ of unity but are on opposite sides of unity. The weighted mean mass bias for Chandra-WL mass bias is $\langle b\rangle=0.12 \pm 0.07$, whereas the weighted mean for Chandra-SaWLens is $\langle b\rangle=$ $-0.11 \pm 0.07$. Some of the difference between these massbias measurements arises from a systematic offset between the WL and SaWLens mass profiles, which is $\approx 10 \pm 5 \%$ at $0.5 \mathrm{Mpc}$, averaged over all 16 clusters with both WL and SaWLens coverage (see Table 6). The remainder of the difference reflects the omission of two clusters with relatively large SaWLens-WL mass-ratio factors (A383 and MS 2137) from the Chandra-lensing comparison at $0.5 \mathrm{Mpc}$.

Combining the strong lensing with weak lensing (SaWLens) reduces the mass dispersion relative to Chandra, compared with WL alone. Dispersion in the mass ratio at $0.5 \mathrm{Mpc}$ for the 

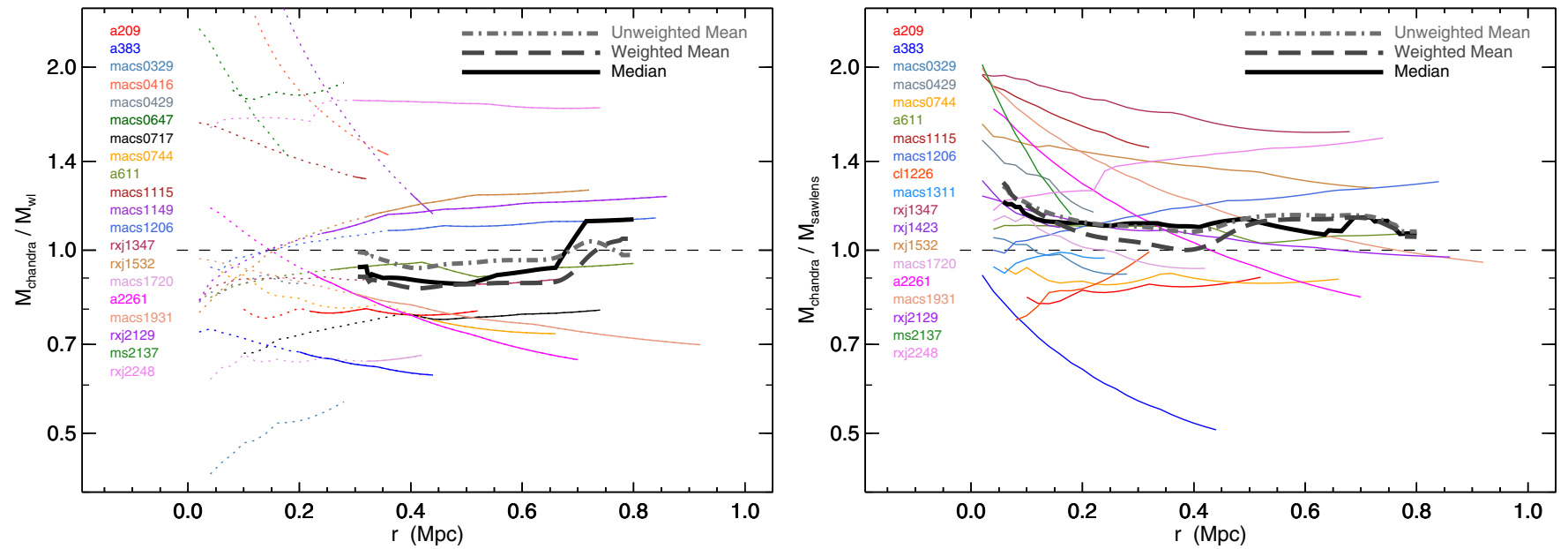

Figure 7. Average ratios of JACO HSE mass profiles from Chandra data to the CLASH weak-lensing (left panel) and strong+weak-lensing (right panel) profiles. Thick solid lines show the median ratios. Long-dashed thick lines show weighted means. Dot-dashed thick lines show unweighted means. Short-dashed lines indicate the locus of equality. Lists at the left show the clusters represented, whose best-fit profile ratios are given by the thin lines. Dotted extensions to those lines in the left panel show extrapolations inside of $1^{\prime}$, where the best-fitting NFW models to the WL data are not well constrained and are not used to compute means or medians. (A color version of this figure is available in the online journal.)
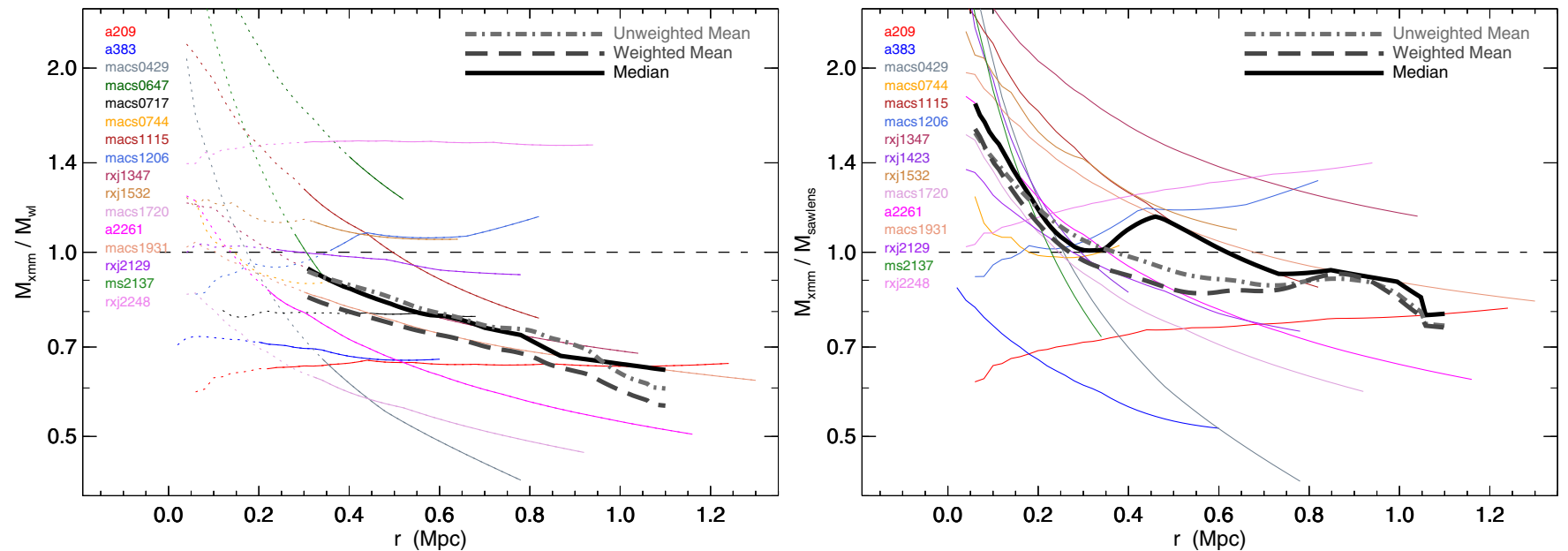

Figure 8. Average ratios of JACO HSE mass profiles from XMM data to the CLASH weak-lensing (left panel) and CLASH strong+weak-lensing (right panel) profiles. Thick solid lines show the median ratios. Long-dashed thick lines show weighted means. Dot-dashed thick lines show unweighted means. Short-dashed lines indicate the locus of equality. Lists at the left show the clusters represented, whose best-fit profile ratios are given by the thin lines. Dotted extensions to those lines in the left panel show extrapolations inside of $1^{\prime}$, where the best-fitting NFW models to the WL data are not well constrained and are not used to compute means or medians.

(A color version of this figure is available in the online journal.)

Chandra-SaWLens cluster set is $\lesssim 20 \%$ (see $\sigma_{\ln f}$ column of Table 6), indicating an intrinsic scatter of $\lesssim 8 \%$ (see $\sigma_{\ln b}^{\text {intrinsic }}$ column) after accounting for uncertainties in the mass-profile measurements. This quantity is not too different from what might be expected from the intrinsic scatter induced by projected structure along the line of sight.

As noted in Umetsu et al. (2014), the predictions of Rozo et al. (2014) for the mass enclosed within $R_{500}$ do not significantly differ from the WL masses in Umetsu et al. (2014): $\left\langle M_{\mathrm{Rozo}} / M_{\mathrm{WL}}\right\rangle=1.13 \pm 0.10$. (To avoid aperture-induced errors, the WL mass was computed inside the same radius.) Since Rozo et al. (2014) estimate a systematic uncertainty in their mass prediction (based on their X-ray luminosity estimates), there is no tension. The WL (and SaWLens) masses are what Rozo et al. would have expected for CLASH clusters based on their X-ray luminosities. Furthermore, the lensing-HSE X-ray mass ratio of $\sim 1$ is similar to what other groups have derived for Chandra HSE mass-WL comparisons (Mahdavi et al. 2013; Newman et al. 2013; Israel et al. 2014). For example, Mahdavi et al. (2013) see this very same effect but apply a correction to the Chandra profiles to bring them into agreement with $X M M$. That work found a difference in the Chandra-XMM mass offset between non-cool-core and cool-core clusters, in that their cool-core clusters had X-ray/WL mass ratios that were constant with radius while their non-cool-core clusters had declining profiles like those in Figure 8. We do not see such a difference. Also, when we add strong-lensing constraints to the lensing mass estimates, scatter in the X-ray/lensing mass relation decreases, indicating that the strong-lensing constraints improve the relation.

\section{XMM-LENSING COMPARISON}

As one might anticipate from Figure 3, our XMM mass-bias measurements are less well behaved than those for Chandra. Figure 8 shows how our XMM JACO mass profiles compare with those from the CLASH WL and SaWlens analyses. There is a strong radial trend, with $X M M$ masses tending to exceed 

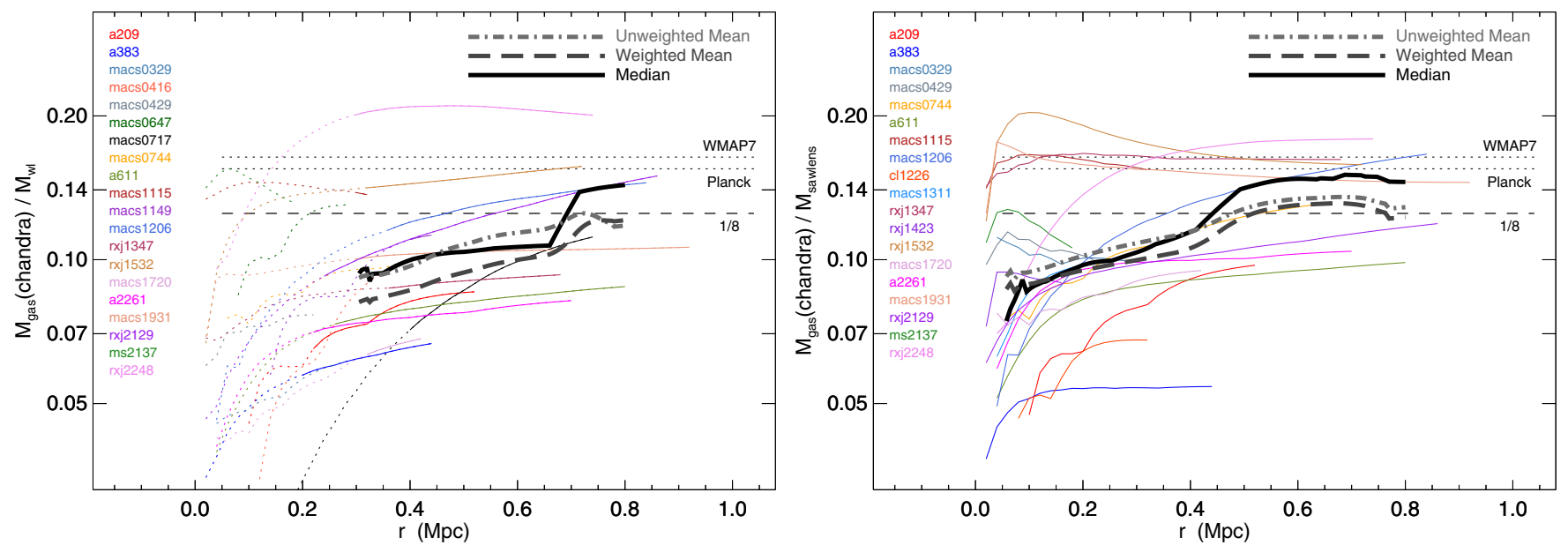

Figure 9. Average ratios of Chandra gas-mass profiles to the CLASH weak-lensing (left panel) and strong+weak-lensing $M_{\mathrm{gas}}$ (right panel) profiles. Thick solid lines show the median ratios. Long-dashed thick lines show weighted means. Dot-dashed thick lines show unweighted means. Level short-dashed lines indicate a gas fraction of 1/8. Level dotted lines show the cosmic baryon mass fractions found by Planck and WMAP. Lists at the left show the clusters represented, whose best-fit profile ratios are given by the thin lines. Dotted extensions to those lines in the left panel show extrapolations inside of $1^{\prime}$, where the best-fitting NFW models to the WL data are not well constrained and are not used to compute means or medians.

(A color version of this figure is available in the online journal.)
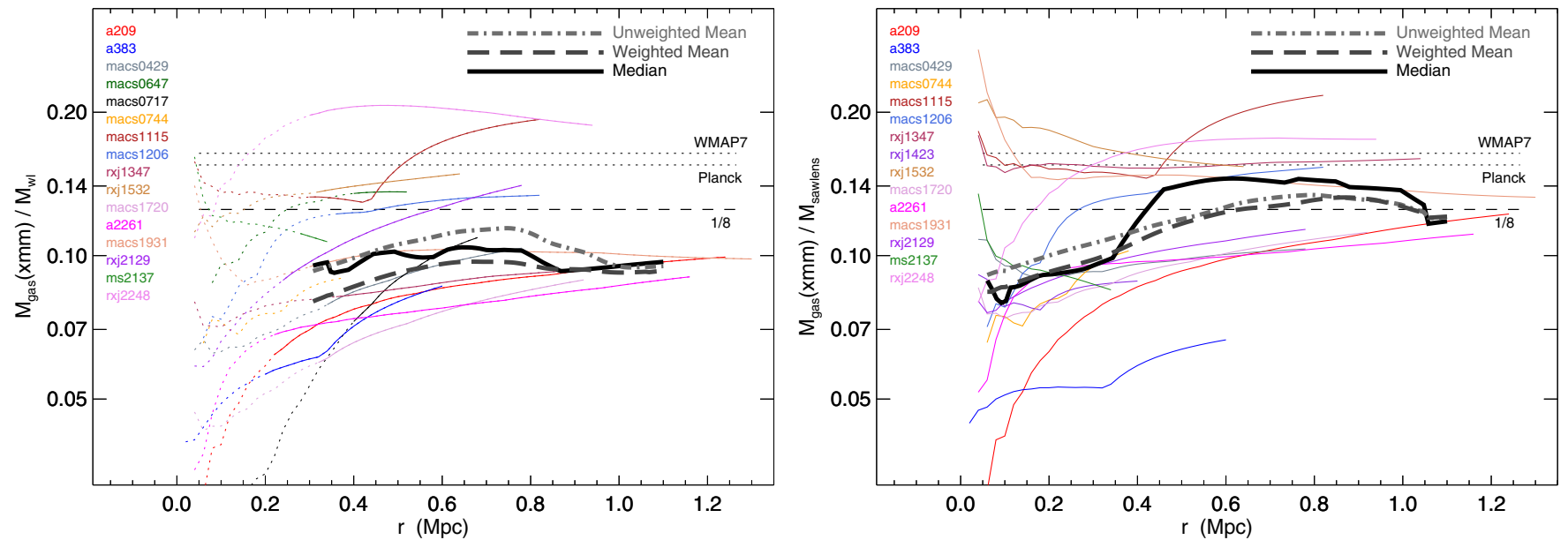

Figure 10. Average ratios of XMM gas-mass profiles to the CLASH weak-lensing (left panel) and strong+weak-lensing (right panel) profiles. Thick solid lines show the median ratios. Long-dashed thick lines show weighted means. Dot-dashed thick lines show unweighted means. Level short-dashed lines indicate a gas fraction of 1/8. Level dotted lines show the cosmic baryon mass fractions found by Planck and WMAP. Lists at the left show the clusters represented, whose best-fit profile ratios are given by the thin lines. Dotted extensions to those lines in the left panel show extrapolations inside of $1^{\prime}$, where the best-fitting NFW models to the WL data are not well constrained and are not used to compute means or medians.

(A color version of this figure is available in the online journal.)

lensing masses at small radii and to fall below them at large radii. Also, the dispersions in mass bias for the $X M M$ comparisons are greater than for the Chandra comparisons. The weighted mean mass bias for $X M M-\mathrm{WL}$ at $0.5 \mathrm{Mpc}$ is $\langle b\rangle=0.24 \pm 0.05$ and for $X M M$-SaWlens is $\langle b\rangle=0.18 \pm 0.08$. It is smaller at $0.8 \mathrm{Mpc}$, because some of the more highly biased clusters have dropped out of the eight-cluster average at this radius, but this reversal of the overall trend appears to be a statistical fluke that does not represent the overall trend evident in the mass-profile ratios of individual clusters. At $1.1 \mathrm{Mpc}$, the decline of the averages has resumed, but there are only three clusters remaining in the $X M M$ sample at this radius, for which $\langle b\rangle=0.44 \pm 0.05$ for $X M M-\mathrm{WL}$ and $\langle b\rangle=0.25 \pm 0.07$ for $X M M-$ SaWlens.

Our results may seem to disagree with the general conclusions of Zhang et al. (2010), who find only a small mass discrepancy between WL mass and XMM HSE masses. However, our $\mathrm{X}$-ray $X M M$ HSE masses agree quite well with theirs for the
Table 7

LOCUSS (Zhang)-XMM Comparison

\begin{tabular}{lcccc}
\hline \hline Name & $\begin{array}{c}\text { Zhang } M_{2500} \\
\left(10^{14} M_{\odot}\right)\end{array}$ & $\begin{array}{c}\text { Zhang } r_{S} \\
(\mathrm{Mpc})\end{array}$ & $\begin{array}{c}\text { CLASH } \\
\left(10^{14} M_{\odot}\right)\end{array}$ & $\begin{array}{c}\text { CLASH } r_{S} \\
(\mathrm{Mpc})\end{array}$ \\
\hline A209 & $1.95 \pm 0.55$ & $0.208 \pm 0.014$ & $1.73 \pm 0.11$ & $0.22 \pm 0.005$ \\
$\mathrm{~A} 383$ & $1.61 \pm 0.48$ & $0.127 \pm 0.007$ & $1.61 \pm 0.07$ & $0.15 \pm 0.005$ \\
$\mathrm{~A} 2261$ & $2.77 \pm 0.75$ & $0.266 \pm 0.026$ & $2.78 \pm 0.09$ & $0.32 \pm 0.006$ \\
$\mathrm{R} 2129$ & $1.75 \pm 0.52$ & $0.166 \pm 0.009$ & $1.96 \pm 0.64$ & $0.24 \pm 0.004$ \\
\hline
\end{tabular}

four CLASH clusters in common (see Table 7). We also note similar agreement for our XMM HSE mass estimates for these four clusters and the $X M M$ estimates derived using independent analyses in Planck Collaboration et al. (2013b). The fact that we derive very similar $X M M$ HSE masses implies that our Xray analyses are compatible with previous studies and the WL masses used in Zhang et al. and that the Planck collaboration 
must be somewhat lower than the lensing masses for CLASH clusters estimated by Umetsu et al. (2014) and Merten et al. (2014).

In Section 9 we discuss the implications of these findings for the discrepancy between the cosmological parameters inferred from the Planck analysis of primary CMB fluctuations and the Planck SZ cluster counts.

\section{GAS MASS-LENSING COMPARISONS}

At large radii, the total gas mass of a cluster is a potentially accurate proxy for its total mass. Our CLASH-X profile comparisons support this notion (Figures 9 and 10). The fact that these mass-profile ratios generally flatten out near $\approx 0.125=1 / 8$ at $\sim 0.5 \mathrm{Mpc}$ (i.e., near $r_{2500}$ in these clusters) indicates that $8 M_{\text {gas }}$ is a good mass proxy there, with only a small amount of bias (see also Vikhlinin et al. (2003); Allen et al. (2004, 2002); Mantz et al. (2008); Ettori et al. (2009)). The flatness of the $M_{\text {gas }} / M_{\text {SaWLens }}$ profiles is particularly striking in this regard, especially for the Chandra comparison, since the formulae JACO uses to fit the gas density and dark matter density profiles are completely different, and yet many of the ratio profiles are remaining within $\sim 10 \%$ of constancy from $\lesssim 0.5 \mathrm{Mpc}$ to beyond $\gtrsim 1.0 \mathrm{Mpc}$. If this constancy in the ratio of gas mass to total mass continues to larger radii, it bodes well for the use of SZ observations to measure total-mass profiles using gas mass as a proxy.

The bottom part of Table 6 lists average mass-ratio factors for comparisons of $M_{\text {gas }}$ to lensing masses. At $0.5 \mathrm{Mpc}$ for Chandra, the weighted means imply $\left\langle f_{\text {gas }}\right\rangle=0.094 \pm 0.007$ relative to $\mathrm{WL}$ and $\left\langle f_{\text {gas }}\right\rangle=0.124 \pm 0.010$ relative to SaWLens. At $0.5 \mathrm{Mpc}$ for $X M M$, we find $\left\langle f_{\text {gas }}\right\rangle=0.093 \pm 0.006$ relative to $\mathrm{WL}$ and $\left\langle f_{\text {gas }}\right\rangle=0.117 \pm 0.011$ relative to SaWLens. As radii rise to $1.1 \mathrm{Mpc}$, these gas fractions remain relatively constant. Apparent differences between $\left\langle f_{\text {gas }}\right.$ values for Chandra and $X M M$ do not arise from differences in the gas-mass measurements, because those are virtually identical (see Figure 1). Instead, they come from scatter in the lensing masses and the fact that the cluster-comparison sets for Chandra and XMM differ.

Notably, the intrinsic scatter between $M_{\text {gas }}$ and lensing mass is smaller for SaWLens than for WL, in alignment with our finding for the comparisons of Chandra HSE masses with lensing masses. Together, these findings confirm that the SaWLens analysis reduces the intrinsic scatter between true spherical mass and spherical mass inferred from lensing, compared with the intrinsic scatter inferred from the WL data alone. Furthermore, the intrinsic scatter for $M_{\text {gas }}$ relative to SaWLens at $0.8 \mathrm{Mpc}$ is only $\approx 12 \%$ for both $X M M$ and Chandra, indicating that both $8 M_{\text {gas }}$ and SaWLens mass are low-scatter proxies for true spherical mass, with minimal bias.

At $f_{\text {gas }}$ of 0.125 , the hot gas in these massive clusters accounts for most but not all of the universal baryon budget of 0.155 (Planck Collaboration et al. 2013a). If the contents of clusters are, on average, representative of the rest of the universe, the remainder is likely to be largely made up of stars. Clusters could be preferentially baryon-poor compared to other regions in the universe, although depletions of more than $10 \%$ seem theoretically unlikely. However, a stellar mass fraction of $3 \%$ is somewhat greater than is typically estimated for massive clusters, even when intracluster light is taken into account (Gonzalez et al. 2013). But uncertainties in the BCG initial mass function and the systematic uncertainties in our mass estimates are still too great to conclude that these studies are missing much of the stellar mass.

\section{IMPLICATIONS FOR THE PLANCK CLUSTER-MASS DISCREPANCY}

In order to resolve the Planck cluster-mass discrepancy (Planck Collaboration et al. 2013c), that is, the tension between the number of clusters Planck finds via the SZ signal and the number of clusters predicted from the cosmological parameters inferred from the primary $\mathrm{CMB}$ power spectrum, a mass bias of $\left\langle b_{X M M}\right\rangle \sim 0.4$ (Planck Collaboration et al. 2013c) is needed. Our comparison between $X M M$ and lensing masses for the CLASH sample does indeed find significant mass bias in $X M M$ hydrostatic mass measurements. However, assigning a single number to that bias is difficult, because it depends on both the radius within which mass is measured and the lensing data (i.e., WL or SaWLens) used to determine the magnitude of that bias. Additionally, the $X M M$-WL bias of $\left\langle b_{X M M}\right\rangle \sim 0.44$ at $1.1 h_{70}^{-1} \mathrm{Mpc}$ is sufficient to account for the entire discrepancy, but is based on averaging over only three CLASH clusters.

Alternatively, one can extrapolate the best-fitting JACO X-ray mass profiles to radii larger than the range of the hydrostatic model. All the CLASH clusters with lensing data can then be included in the averages, but at the expense of statistical significance and perhaps also additional systematic biases. With these caveats, we present in Table 5 the mass bias factors obtained from extrapolations out to $r_{500}$, using the $r_{500}$ determined from the same data set. That procedure further amplifies any mass bias present at a fixed radius, because of the aperture-induced covariance effect, and results in $\left\langle b_{X M M-\mathrm{WL}}\right\rangle=$ $0.44 \pm 0.06$ and $\left\langle b_{X M M-\text { SaWlens }}\right\rangle=0.47 \pm 0.08$. The $\chi_{v}^{2}$ values for these averages are unacceptably large, indicating that we have stretched most of the CLASH X-ray data beyond the limits of reliability, meaning that the formal uncertainties on these mass-bias values are too small. However, the overall trend does appear to be real.

Our results therefore are in alignment with the finding of the Weighing the Giants (WtG) collaboration (von der Linden et al. 2014), that the default mass calibration adopted by the Planck team $(\langle b\rangle=0.2)$ underestimates the true masses at large radii. We approached this question somewhat differently from the $\mathrm{WtG}$ team, in that we are deriving HSE masses directly from the $X M M$ data for our sample of CLASH clusters, and because our mass profiles benefit from additional information: Merten et al. (2014) utilize strong-lensing constraints on the weak shear profiles in the case of SaWLens, and Umetsu et al. (2014) include magnification constraints in the case of CLASH-WL. These new lensing masses are consistent with the lensing masses derived by $\mathrm{WtG}$ based on shear alone; our analysis of the $\mathrm{X}$ ray observations for the same clusters shows that the XMM HSE masses, derived directly (and not from scaling relations), are also consistent with $\langle b\rangle$ considerably larger than 0.2 , for either WL or SaWLens masses as surrogates for the gravitating masses.

\section{CONCLUSIONS}

The following are our primary findings.

1. Chandra and XMM measurements of electron density and enclosed gas mass as functions of radius are highly consistent with one another, indicating that any differences in HSE masses inferred from X-ray observations arise from differences in gas-temperature measurements (Section 4.1).

2. Gas temperatures measured in clusters by $X M M$ and Chandra are consistent with one another at $\sim 100 \mathrm{kpc}$ radii, but $X M M$ temperatures systematically decline relative to 
Chandra temperatures as the radius of the temperature measurement increases (Section 4.2). Plausible contributions to this apparent temperature difference are large-angle scattering of soft X-ray photons in excess of that amount expected from the standard $X M M$ PSF correction, a radial variation in the quality of the soft energy calibration of both telescopes, and uncertain vignetting corrections. While we cannot state with finality that the Chandra absolute calibration is better than XMM's, the Chandra-derived cluster HSE mass profiles are significantly more similar in shape and normalization to the CLASH strong- and weak-lensing profiles presented in Umetsu et al. (2014) and Merten et al. (2014). We plan and encourage future work in cross-comparison of $X M M$ and Chandra cluster analyses to unlock the full potential of the investment of both observatories.

3. We present the CLASH-X mass-profile comparisons in the form of circular-velocity profiles, because sharing and comparing results in that form has several advantages: Mass profiles provided in terms of $v_{\text {circ }}\left(\theta_{r}\right)$ are independent of cosmological assumptions. Plots of $v_{\text {circ }}(r)$ span much less dynamic range than $M_{r}$ plots, making systematic differences among profiles more apparent. The scale radius $r_{\mathrm{s}}$ and maximum circular velocity $v_{\max }$ of a halo do not change if the halo does not change, whereas its mass, radius, and concentration continually increase if those quantities are defined with respect to a spherical-overdensity threshold $\Delta$. A halo's value of $v_{\max }$ is therefore a more general indicator of its properties than $M_{\Delta}$, because it is independent of redshift. Furthermore, accurate estimates of $v_{\max }$ can be obtained from information at many different radii, because $v_{\text {circ }}(r)$ curves for NFW profiles are nearly level at the radii of greatest interest, remaining within $2 \%$ of $v_{\max }$ over the interval $1.4 \lesssim r / r_{\mathrm{s}} \lesssim 3.5$

4. Ratios of Chandra HSE mass profiles to CLASH strongand weak-lensing profiles show no obvious radial dependence in the 0.3-0.8 Mpc range. However, the mean mass biases inferred from the WL and SaWLens data are different, with a weighted-mean value at $0.5 \mathrm{Mpc}$ of $\langle b\rangle=0.12$ for the WL comparison and $\langle b\rangle=-0.11$ for the SaWLens comparison.

5. Ratios of XMM HSE mass profiles to CLASH lensing profiles show a pronounced radial dependence in the 0.3-1.0 Mpc range, with a weighted-mean mass bias of value rising to $\langle b\rangle=0.3$ at $1 \mathrm{Mpc}$ for the WL comparison and $\langle b\rangle=0.2$ for the SaWLens comparison.

6. Enclosed gas mass profiles from both Chandra and XMM rise to $\sim 0.125$ times the total-mass profiles inferred from lensing at $\approx 0.5 \mathrm{Mpc}$ and remain constant outside of that radius, indicating that $8 M_{\text {gas }}$ profiles may be a useful proxy for total-mass profiles at $\gtrsim 0.5 \mathrm{Mpc}$ in massive galaxy clusters.

M.D. and A.H. acknowledge the partial support of STScI/NASA award HST-GO-12065.07-A and NASA award NNX13AI41G. M.D. and G.M.V. benefitted from discussions of this work with Jim Bartlett. The Dark Cosmology Centre is funded by the DNRF. A.M. was partially supported through NASA ADAP award NNX12AE45G. S.E. acknowledges the financial contribution from contracts ASI-INAF I/023/05/0 and I/088/06/0. The work of L.A.M. and J.M. was carried out at Jet Propulsion Laboratory, California Institute of Technology, under a contract with NASA. M.M. acknowledges financial contribution from the agreement ASI/INAF I/023/12/0 and from the project INFN PD51. Support for A.Z. is provided by NASA through a Hubble Fellowship grant HST-HF-51334.01-A awarded by STScI.

$$
\text { Facilities: CXO, XMM, HST }
$$

\section{REFERENCES}

Allen, S. W., Schmidt, R. W., Ebeling, H., Fabian, A. C., \& van Speybroeck, L. 2004, MNRAS, 353, 457

Allen, S. W., Schmidt, R. W., \& Fabian, A. C. 2002, MNRAS, 334, L11

Arnaud, M., Pointecouteau, E., \& Pratt, G. W. 2007, A\&A, 474, L37

Arnaud, M., Pratt, G. W., Piffaretti, R., et al. 2010, A\&A, 517, A92

Balestra, I., Vanzella, E., Rosati, P., et al. 2013, A\&A, 559, L9

Battye, R. A., \& Moss, A. 2014, PhRvL, 112, 051303

Becker, M. R., \& Kravtsov, A. V. 2011, ApJ, 740, 25

Carter, J. A., \& Read, A. M. 2007, A\&A, 464, 1155

Cavagnolo, K. W., Donahue, M., Voit, G. M., \& Sun, M. 2009, ApJS, 182, 12

Coe, D., Umetsu, K., Zitrin, A., et al. 2012, ApJ, 757, 22

Coe, D., Zitrin, A., Carrasco, M., et al. 2013, ApJ, 762, 32

Croston, J. H., Arnaud, M., Pointecouteau, E., \& Pratt, G. W. 2006, A\&A, 459, 1007

Croston, J. H., Pratt, G. W., Böhringer, H., et al. 2008, A\&A, 487, 431

Dalal, N., Hennawi, J. F., \& Bode, P. 2005, ApJ, 622, 99

David, L. P., Jones, C., \& Forman, W. 1995, ApJ, 445, 578

Dolag, K., Vazza, F., Brunetti, G., \& Tormen, G. 2005, MNRAS, 364, 753

Ebeling, H., Edge, A. C., \& Henry, J. P. 2001, ApJ, 553, 668

Ebeling, H., Ma, C.-J., \& Barrett, E. 2014, ApJS, 211, 21

Eichner, T., Seitz, S., Suyu, S. H., et al. 2013, ApJ, 774, 124

Ettori, S. 2013, MNRAS, 435, 1265

Ettori, S., De Grandi, S., \& Molendi, S. 2002, A\&A, 391, 841

Ettori, S., Gastaldello, F., Leccardi, A., et al. 2010, A\&A, 524, A68

Ettori, S., Morandi, A., Tozzi, P., et al. 2009, A\&A, 501, 61

Evrard, A. E. 1990, ApJ, 363, 349

Evrard, A. E., Bialek, J., Busha, M., et al. 2008, ApJ, 672, 122

Evrard, A. E., Metzler, C. A., \& Navarro, J. F. 1996, ApJ, 469, 494

Finoguenov, A., Reiprich, T. H., \& Böhringer, H. 2001, A\&A, 368, 749

Gioia, I. M., Maccacaro, T., Schild, R. E., et al. 1990, ApJS, 72, 567

Gonzalez, A. H., Sivanandam, S., Zabludoff, A. I., \& Zaritsky, D. 2013, ApJ, 778,14

Gott, J. R., III, \& Gunn, J. E. 1971, ApJL, 169, L13

Graur, O., Rodney, S. A., Maoz, D., et al. 2014, ApJ, 783, 28

Gruen, D., Brimioulle, F., Seitz, S., et al. 2013, MNRAS, 432, 1455

Hamann, J., \& Hasenkamp, J. 2013, JCAP, 10, 044

Henry, J. P., \& Arnaud, K. A. 1991, ApJ, 372, 410

Hickox, R. C., \& Markevitch, M. 2007, ApJL, 661, L117

Hoekstra, H. 2001, A\&A, 370, 743

Hoekstra, H. 2003, MNRAS, 339, 1155

Horner, D. J., Mushotzky, R. F., \& Scharf, C. A. 1999, ApJ, 520, 78

Israel, H., Reiprich, T. H., Erben, T., et al. 2014, A\&A, 564, A129

Kaiser, N., \& Squires, G. 1993, ApJ, 404, 441

King, L., \& Corless, V. 2007, MNRAS, 374, L37

Leccardi, A., \& Molendi, S. 2008, A\&A, 486, 359

Mahdavi, A., Hoekstra, H., Babul, A., et al. 2007, ApJ, 664, 162

Mahdavi, A., Hoekstra, H., Babul, A., et al. 2013, ApJ, 767, 116

Mantz, A., Allen, S. W., Ebeling, H., \& Rapetti, D. 2008, MNRAS, 387, 1179

Mantz, A., Allen, S. W., Rapetti, D., \& Ebeling, H. 2010, MNRAS, 406, 1759

Markevitch, M., Bautz, M. W., Biller, B., et al. 2003, ApJ, 583, 70

McLaughlin, D. E. 1999, AJ, 117, 2398

Medezinski, E., Umetsu, K., Nonino, M., et al. 2013, ApJ, 777, 43

Meneghetti, M., Rasia, E., Vega, J., et al. 2014, ApJ, submitted (arXiv:1404.1384)

Merten, J., Meneghetti, M., Postman, M., et al. 2014, ApJ, submitted (arXiv:1404.1376)

Metzler, C. A., White, M., \& Loken, C. 2001, ApJ, 547, 560

Monna, A., Seitz, S., Greisel, N., et al. 2014, MNRAS, 438, 1417

Nagai, D., Kravtsov, A. V., \& Vikhlinin, A. 2007, ApJ, 668, 1

Navarro, J. F., Frenk, C. S., \& White, S. D. M. 1997, ApJ, 490, 493

Nelson, K., Lau, E. T., Nagai, D., Rudd, D. H., \& Yu, L. 2014, ApJ, 782, 107

Nevalainen, J., David, L., \& Guainazzi, M. 2010, A\&A, 523, A22

Newman, A. B., Treu, T., Ellis, R. S., et al. 2013, ApJ, 765, 24

Okabe, N., Zhang, Y.-Y., Finoguenov, A., et al. 2010, ApJ, 721, 875

Patel, B., McCully, C., Jha, S. W., et al. 2014, ApJ, 786, 9

Pierre, M., Valtchanov, I., Altieri, B., et al. 2004, JCAP, 09, 011

Planck Collaboration, Ade, P. A. R., Aghanim, N., et al. 2013a, A\&A, in press (arXiv:1303.5076) 
Planck Collaboration, Ade, P. A. R., Aghanim, N., et al. 2013b, A\&A, 550, A129

Planck Collaboration, Ade, P. A. R., Aghanim, N., et al. 2013c, A\&A, 550, A131

Pointecouteau, E., Arnaud, M., Kaastra, J., \& de Plaa, J. 2004, A\&A, 423, 33

Postman, M., Coe, D., Benítez, N., et al. 2012, ApJS, 199, 25

Rasia, E., Meneghetti, M., Martino, R., et al. 2012, NJPh, 14, 055018

Reiprich, T. H., Basu, K., Ettori, S., et al. 2013, SSRv, 177, 195

Rosati, P., della Ceca, R., Norman, C., \& Giacconi, R. 1998, ApJL, 492, L21

Rozo, E., Bartlett, J. G., Evrard, A. E., \& Rykoff, E. S. 2014, MNRAS, 438,78

Schellenberger, G., Reiprich, T. H., Lovisari, L., Nevalainen, J., \& David, L. 2014, A\&A, submitted (arXiv:1404.7130)

Sijacki, D., \& Springel, V. 2006, MNRAS, 371, 1025

Tyson, J. A., Wenk, R. A., \& Valdes, F. 1990, ApJL, 349, L1
Umetsu, K., Medezinski, E., Nonino, M., et al. 2012, ApJ, 755, 56

Umetsu, K., Medezinski, E., Nonino, M., et al. 2014, ApJ, in press (arXiv:1404.1375)

Vikhlinin, A., Burenin, R. A., Ebeling, H., et al. 2009, ApJ, 692, 1033

Vikhlinin, A., Kravtsov, A., Forman, W., et al. 2006, ApJ, 640, 691

Vikhlinin, A., Voevodkin, A., Mullis, C. R., et al. 2003, ApJ, 590, 15

von der Linden, A., Mantz, A., Allen, S. W., et al. 2014, MNRAS, 443, 1973

Wyman, M., Rudd, D. H., Vanderveld, R. A., \& Hu, W. 2014, PhRvL, 112, 051302

Xu, H., Jin, G., \& Wu, X.-P. 2001, ApJ, 553, 78

Zhang, Y.-Y., Finoguenov, A., Böhringer, H., et al. 2007, A\&A, 467, 437

Zhang, Y.-Y., Okabe, N., Finoguenov, A., et al. 2010, ApJ, 711, 1033

Zheng, W., Postman, M., Zitrin, A., et al. 2012, Natur, 489, 406

Zitrin, A., Moustakas, J., Bradley, L., et al. 2012a, ApJL, 747, L9

Zitrin, A., Rosati, P., Nonino, M., et al. 2012b, ApJ, 749, 97 